\title{
Anomalies in the detection of change: When changes in sample size are mistaken for changes in proportions
}

\author{
Klaus Fiedler ${ }^{1}$ - Yaakov Kareev ${ }^{2}$ - Judith Avrahami ${ }^{2}$ - Susanne Beier ${ }^{1}$. \\ Florian Kutzner $^{1}$ • Mandy Hütter ${ }^{1}$
}

Published online: 16 July 2015

(C) Psychonomic Society, Inc. 2015

\begin{abstract}
Detecting changes, in performance, sales, markets, risks, social relations, or public opinions, constitutes an important adaptive function. In a sequential paradigm devised to investigate detection of change, every trial provides a sample of binary outcomes (e.g., correct vs. incorrect student responses). Participants have to decide whether the proportion of a focal feature (e.g., correct responses) in the population from which the sample is drawn has decreased, remained constant, or increased. Strong and persistent anomalies in change detection arise when changes in proportional quantities vary orthogonally to changes in absolute sample size. Proportional increases are readily detected and nonchanges are erroneously perceived as increases when absolute sample size increases. Conversely, decreasing sample size facilitates the correct detection of proportional decreases and the erroneous perception of nonchanges as decreases. These anomalies are however confined to experienced samples of elementary raw events from which proportions have to be inferred inductively. They disappear when sample proportions are described as percentages in a normalized probability format. To explain these challenging findings, it is essential to understand the inductivelearning constraints imposed on decisions from experience.
\end{abstract}

Keywords Decision making · Judgment · Inductive reasoning $\cdot$ Memory

Klaus Fiedler

kf@psychologie.uni-heidelberg.de

University of Heidelberg, Heidelberg, Germany

2 Hebrew University of Jerusalem, Jerusalem, Israel

\section{Introduction}

One of the most intricate demands imposed on inference making is the joint consideration of intensional and extensional information, or quality and quantity. How does the persuasiveness of communication jointly depend on argument strength and on the number of arguments? How do consumer evaluations reflect the quality and the exposure frequency of a brand? How does the impact of a scientific study depend on validity and citation frequency?

\section{Confusion of intensional and extensional information}

Empirical findings from various research paradigms converge in demonstrating distinct anomalies in quantitative judgments, reflecting the confusion of intensional and extensional information. When judgment or decision problems call for the assessment of proportional quantities - such as proportions of correct responses, acceptance rates, or winning outcomes in lotteries - the estimated proportion of a focal feature is systematically biased by its absolute frequency. Sample size intrudes into the assessment of a proportion within the sample (Estes, 1976). For example, teachers' estimates of students' correct response rates are biased toward the frequency of responses they have provided (Fiedler, Walther, Freytag, and Plessner, 2002). Frequently presented statements tend to be considered true (Dechêne, Stahl, Hansen, and Wänke, 2010). Majorities and large categories appear more positive than equivalent minorities and small categories (Hamilton \& Gifford, 1976). Or, the impact of the same rate of feedback or reinforcement increases with absolute number of learning trials (Allan \& Jenkins, 1983; Perales, Catena, Shanks, \& González, 2005; Shanks \& Dickinson, 1991). Specifically, given a constant proportion $P=F_{\text {large }} / N_{\text {large }}=F_{\text {small }} / N_{\text {small }}$, 
with $F_{\text {large }}$ being the frequency of the focal feature in a large sample of size $N_{\text {large }}$ and $F_{\text {small }}$ being the frequency of the focal feature in a small sample of size $N_{\text {small }}, F_{\text {large }} / N_{\text {large }}$ is experienced as larger than $F_{\text {small }} / N_{\text {small }}$.

Different names have been given to this phenomenon in diverse paradigms. In decision research, the preference for a lottery bowl that offers 10 winning chances (or even only 9) out of 100 over a smaller lottery bowl offering a chance of 1 out of 10 has been termed the ratio bias (Denes-Raj, Epstein, $\&$ Cole, 1995). The larger number of winning opportunities (in the numerator of the ratio) seems to dominate the less apparent disadvantage of the higher set size of the larger bowl (the denominator). A more elaborate account is offered by Reyna and Brainerd's (2008) denominator neglect, which is not confined to very small proportions and highly unequal sample sizes. For instance, children prefer a lottery of 5 winning balls out of 10 to 3 winning balls out of 6 .

In quantitative estimation tasks, too, an analogous samplesize bias was found (Price, 2001; Price, Smith, \& Lench, 2006; Smith \& Price, 2010). Estimates of the average of a set of numbers increase when set size increases but the objective average is held constant, showing that the phenomenon generalizes beyond frequency judgments. Carryover effects from sample size to proportion estimates may remind one of numerical magnitude priming (Oppenheimer, LeBoeuf, \& Brewer, 2008). In social psychology, an increasing positive evaluation after repeated stimulus presentation is called mere-exposure effect (Zajonc, 2001). The analogous tendency to prefer the more familiar (frequently encountered) stimulus in a pair has been called fluency heuristic (Hertwig, Herzog, Schooler, \& Reimer, 2008).

In contingency assessment, when the task is to assess the difference between two proportions, $\Delta=F_{\text {large }} / N_{\text {large }}-F_{\text {small }} /$ $N_{\text {small, }}$, an illusory correlation arises because the proportion $F_{\text {large }} / N_{\text {large }}$ observed in a larger sample is subjectively different from the same proportion $F_{\text {small }} / N_{\text {small }}$ observed in a smaller sample. In different literatures, this phenomenon is known as infrequency-based illusory correlations (Fiedler, 2000a, 2000b; Hamilton \& Gifford, 1976) or as density bias (Allan \& Jenkins, 1983; Perales et al., 2005; Shanks \& Dickinson, 1991). By analogy, a confirmation bias or auto-verification effect in hypothesis testing (Fiedler, Walther, \& Nickel, 1999) occurs when a focal hypothesis is confirmed at the same relative rate but at a higher absolute frequency than an alternative hypothesis.

The intrusion of sample size into proportional judgments appears in so many paradigms linked to so many theoretical ideas that it cannot be supposed to reflect a single cognitive algorithm. In simulation studies, indeed, the depicted biases can be explained by exemplar-based (E. R. Smith, 1991) and category abstraction models (Fiedler, Freytag, \& Meiser, 2009), tutored (Kutzner, Freytag, Vogel, \& Fiedler, 2008) and untutored learning (Fiedler, 1996), recursive (Van Rooy,
Van Overwalle, Vanhoomissen, Labiouse, \& French, 2003) and feedforward algorithms (Fiedler, 1996), symbolic and subsymbolic mechanisms (Smolensky, 1988), gist encoding (Reyna \& Brainerd, 2008), or regression in noisy transmission (Fiedler \& Krueger, 2012; Hilbert, 2012; Kutzner, Vogel, Freytag, \& Fiedler, 2011; Moore \& Healy, 2008).

\section{Detection of change}

The multitude of models that predict the subjective inequality of $F_{\text {large }} / N_{\text {large }}$ and $F_{\text {small }} / N_{\text {small }}$ prevents us from seeking a unique underlying mechanism. Large and small samples differ in so many confounded ways - unequal learning opportunities and memory load, sampling error and uncertainty, variability and density of representations - that we refrain from an attempt to explain the entire phenomenon at the mechanistic level. We do believe, however, that a functional-level analysis of the phenomenon's origins and boundary conditions, within a detection-of-change paradigm, leads to a deeper understanding.

Change assessment serves a major adaptive function in a probabilistic world with ever changing risks, challenges, and opportunities. Humans must be sensitive to changes in power, norms, social relations, health threats, and political and economic strategies. Marketers must detect sales changes; politicians must be alerted to changing acceptance rates of political programs; teachers must update evaluations in students' performance over time; and, scientists must monitor and revise theoretical beliefs in the light of new empirical findings.

An essential characteristic of all these real-life examples is that the task calls for inferences of latent proportions that are not amenable to direct observation. Going beyond the mere assessment of given sample proportions, change detection involves inferences from restricted samples to a latent universe. Marketers infer changes in the latent market, not just in an observed statistic. Politicians, teachers, and scientists are not only interested in changes in local samples of audiences, lessons, or studies, they are also interested in latent political trends, student abilities, or validity of theories. Accurately assessing sample proportions is necessary but not sufficient for the accurate inference of true changes in the latent reality.

Though essential and representative of what marketers, politicians, teachers, and scientists do all the time, this inferential aspect of change detection has been hardly studied systematically. Notable exceptions might be found in prior work on regime shifts (Massey \& Wu, 2005), change detection in nonstationary systems (Brown \& Steyvers, 2009; Chinnis \& Peterson, 1970) and in an autocorrelation paradigm (Speekenbrink, Twyman, \& Harvey, 2012), or continuous probability updating (Rapoport, Stein, \& Burkheimer, 1979; Robinson, 1964). However, no prior research has investigated inferences about latent changes in proportions under 
orthogonally manipulated changes in the size of stimulus samples. In such a paradigm, an increasing $n$ may not only affect the subjective assessment of a proportion in the sample but also the readiness to make an inference to a latent reality.

In the sequential paradigm we use to investigate inferences about latent changes, each trial consists of a sample of binary features. The proportion $p$ of a critical feature (e.g., positive outcomes, correct responses) in the population, and hence the proportion $P$ in the sample drawn from the population, varies across trials. Participants have to decide on each trial whether a change has occurred in the "world" from which the previous and the current samples are drawn. Subsets of transitions from $p_{\text {pre }}$ to $p_{\text {curr }}$ involve decreases, increases or no-change. Orthogonal to these $p$-changes, sample size $n$ varies too, such that equal subsets of transitions at each level of $p$-change involve either increases from $n_{\text {pre }}=8$ to $n_{\text {curr }}=16$ or decreases from $n_{\text {pre }}=16$ to $n_{\text {curr }}=8$.

As will be evident soon, this paradigm produces strong anomalies when $n$-changes are pitted against $p$-changes. While it is easy to detect increases in proportions $p$ when absolute sample size $n$ increases too, or to detect decreases in $p$ that come along with decreasing $n$, it is very difficult to detect changes in $p$ that counteract changes in $n$ (i.e., increases in $p$ when $n$ decreases or decreases in $p$ when $n$ increases). However, these strong anomalies turn out to depend on presentation format. They are only observed when proportions are experienced extensionally. They disappear when proportions are described in normalized probability format - quite inconsistent with the often-cited notion that naturally experienced frequency formats serve to reduce cognitive illusions that are obtained with normalized probability formats (Gigerenzer \& Hoffrage, 1995; Hoffrage \& Gigerenzer, 1998; Sloman, Over, Slovak, \& Stibel, 2003). Change detection seems to provide a notable counterexample.

\section{The inability not to be affected by changing sample size}

Why should change detection be prone to such strong anomalies, and why should mode of presentation matter? Why should $p$ and $n$ be confused only when samples are experienced inductively? A functional analysis of the inductive learning task helps to clarify why it is so difficult to assess $p$ independently of $n$.

Functional analysis of the $\mathbf{P}$-assessment task For an example that is concrete and familiar to everybody, imagine a teacher's task to assess changes in various students' proportions of correct responses. When the proportion of a focal outcome (e.g., correct responses out of all student responses) is experienced as a set of elementary raw observations distributed in time or space, increments or decrements have to be added to a growing update of the sample proportion $P$ as new correct or incorrect answer are observed, respectively. The size of the increment or decrement depends on sample size. In a small sample of $n=4$, the increment or decrement for a single new observation on a proportion scale (ranging from $P=0$ to $P=1$ ) is $1 / 4$. Thus, one correct outcome is sufficient to raise the update from $P=.5$ to $P=.75$; two correct outcomes raise $P$ from .5 to 1 . As sample size gets larger (e.g., $n=8$ or $n=16$ ), the updating effect of new observations decreases. In general, the incremental weight of an elementary observation of size $k$ should be $1 / k^{1}$

Therefore, continuous updating requires that $n$ is known beforehand; otherwise, the increment or decrement to be coded for new observations is indeterminate. This necessary condition, however, is neither met in the teacher's real life nor when $n$ varies across trials of a detectionof-change experiment. The size and limits of naturally occurring or dynamically changing samples are hardly ever known. It is practically impossible for a teacher to know in advance the size of all samples for a hundred or more students in diverse classes, often qualified by disciplines and time frames. Moreover, the beginning and the end of particular samples is hard to define, so there is no natural unit for counting elementary observations.

Thus, the size of countless intertwined samples is not determined beforehand. The teacher is not committed to sampling $n_{\mathrm{i}}$ observations from student $i$. Rather, sample size is an emergent result of a dynamic search process that depends on the teacher's current thoughts and strategies, the students' rate of raising hands, and their salience. Facing such a task of assessing proportions in dynamically growing samples, the teacher might adjust the weight given to new observations from $1 / k$ for observation $k$ to $1 /(k+1)$ for observation $k+1$, producing a clear-cut primacy effect (i.e., decreasing weight given to later observations).

To avoid such a primacy bias, unbiased $P$ assessment in spite of variable $n$ would require an updating rule that is too complicated to be realistic. After every new observation $k$, an updated proportional estimate $P_{\mathrm{k}}$ would have to be recomputed as a weighted average of the new observation $k$ (given a weight of $1 / k$ ) and the previous estimate $P_{k-1}$ (given a weight of $(k-1) / k)$. Again, this requires keeping track of the size of all ongoing samples. Alternatively, a complete copy of all sampled information would have to be stored in memory, so that $P$ can be estimated whenever it is needed from an allinclusive exemplar-based memory.

${ }^{1}$ Note that this is only meant as a functional analysis of the difficulty inherent in the encoding of changes in an experienced sample of uncertain size. It need not imply an exhaustive cognitive encoding algorithm that consists of an incremental update for each of $n$ elementary observations. 
Because all these assumptions strike us as unrealistic, we do not believe that incremental coding of a successively experienced sample can be adjusted to variable sample size. A more realistic assumption is that increments or decrements encoded for focal and nonfocal events will remain roughly equal across growing samples, as in a random walk. Correct student answers will cause a similar upward shift and wrong answers a similar downward shift, in the teacher's impression, regardless of how many future student answers can be expected. When the sampling process is stopped at any point, the resulting estimate should be sensitive, at least to some degree, to a summation (rather than averaging) rule, which explains the confound of $p$ and $n$ : Given 8 elementary increments (focal outcomes) and 2 elementary decrements (nonfocal outcomes), the summed updating effect is higher $(8-2=6)$ than when the same proportion is manifested in 4 focal and 1 nonfocal outcomes $(4-1=3){ }^{2}$ Seminal work by Inhelder and Piaget (1958) and Estes (1976) on probability learning and contingency assessment (Shanks, 1985, 1987) testifies to such a summation effect on inductive inference.

\section{Detecting changes occurring successively or simultaneous-} ly Although our functional analysis restricts the expected anomalies to experienced samples that call for the inductive assessment of $P$ from multiple raw observations, it need not be restricted to successive changes over time. Similar anomalies might be expected for changes in simultaneous samples (e.g., presented left and right on the computer screen), provided the presentation is complex or brief enough to prevent an elementwise comparison process. Thus, when a pair of samples is presented on the right and left side of the computer screen, an increase in $\mathrm{p}$ from left to right should be easier to detect when $n$ also increases from left to right, and vice versa.

Support comes from a long tradition of research on contingency assessment (Allan, 1980; Wasserman, Dorner, \& Kao, 1990; White, 2009). Regardless of whether the two proportions compared in a contingency $\Delta=P_{1}-P_{2}$ (e.g., the rate of a disease given the presence vs. absence of a symptom) are presented successively or simultaneously, the well-known density bias shows that an experienced contingency is positive if $P_{1}$ is based on a larger sample than $P_{2}$, even when both proportions are the same. More recent research on pseudocontingencies (Fiedler et al., 2009) provides further support. Indeed, change detection can be conceived as a special kind of a contingency task, involving a comparison of separately assessed sample proportions.

\footnotetext{
${ }^{2}$ As already mentioned, rather than counting all elements, the updating of experienced samples may involve subsets or small clusters of several observations or many other specific encoding algorithms.
}

Format dependency and the description-experience gap Interestingly, the evidence on contingency assessment exhibits format dependency: The anomalies are only obtained when proportions are learned inductively by experience; they disappear when proportions are described as normalized probabilities or statistical summary tables (Hamilton, Dugan, \& Trolier, 1985; Slovic, Monahan, \& MacGregor, 2000).

This format dependency has two notable theoretical implications. First, if the anomalies are restricted to experienced modes and disappear for descriptive modes, the notion of a ratio bias or a denominator neglect per se can hardly provide a sufficient account because ratios or denominators exist independently of presentation modes. Second, the postulated format dependency relates the present research to a prominent topic in current decision-making research, the so-called description-experience gap (Hau, Pleskac, Kiefer, \& Hertwig, 2008; Hertwig, Barron, Weber, \& Erev, 2004; Hertwig \& Erev, 2009). Decisions informed by samples experienced as sequences of raw events have been shown to deviate from decisions informed by samples described in summary statistics. The central finding, which is the focus of pertinent research, is that rare outcomes are underweighted in decisions from experience but overweighted in decisions from description (for a review, see Hertwig \& Erev, 2009).

In the present research, we borrow the descriptionexperience gap as a moderator of anomalies in change detection but we also increase its scope and widen its theoretical status. While previous evidence is largely confined to underand overweighting of rare outcomes, we demonstrate that the catalyst role of experienced format for the production of anomalies in change detection is not restricted to low- $p$ trials. Rather, the inductive experience of sample proportions should produce the predicted anomalies across all levels of $p$.

Moreover, extending the description-experience gap to this new domain affords an effective means of ruling out the possibility that the gap is merely due to sampling error, that is, to the fact that small samples tend to underrepresent rare outcomes (Fox \& Hadar, 2006; Hau et al., 2008; Rakow, Demes, \& Newell, 2008; Ungemach, Chater, \& Stewart, 2009). While this statistical fact could (at least partly) account for underweighting of rare outcomes in the experienced mode, it does not apply to the present paradigm for two reasons. First, as already stated, the anomalies should not be restricted to small- $p$ samples but should also be obtained with higher values of $p$ that are not underrepresented in small samples. Secondly, by using a yoked design, we present the very same samples in descriptive and experience format, rather than comparing descriptions of $p$ in the population with experiences of $P$ in a sample. 


\section{Plan of empirical research}

Summary of predictions The experiments reported below were designed to test the following predictions. The assessment of changes from $p_{\text {pre }}$ to $p_{\text {curr }}$ will depend on sample-size changes from $n_{\text {pre }}$ to $n_{\text {curr }}$ Actual increases $\left(p_{\text {pre }}<p_{\text {curr }}\right)$ will be more easily detected when sample size increases too $\left(n_{\text {pre }}<\right.$ $\left.n_{\text {curr }}\right)$. If sample size decreases $\left(n_{\text {pre }}>n_{\text {curr }}\right)$ while proportions increase ( $p_{\text {pre }}<p_{\text {curr }}$ ), opposing influences of $n$ and $p$ will impair change detection. Conversely, downward changes $\left(p_{\text {pre }}>\right.$ $\left.\mathrm{p}_{\text {curr }}\right)$ will be readily detected if sample size decreases too ( $n$ pre $\left.>n_{\text {curr }}\right)$, but hard to detect when extensity and intensity work against each other (i.e., when $n_{\text {pre }}<n_{\text {curr }}$ ).

On no-change trials, when two successive stimulus samples are drawn from the same population $\left(p_{\text {pre }}=p_{\text {curr }}\right)$, increases in sample size ( $\left.n_{\text {pre }}<n_{\text {curr }}\right)$ would be likely mistaken as increases in $p$, yielding a substantial rate of upward false alarms. Conversely, decreases in sample size $\left(n_{\text {pre }}>n_{\text {curr }}\right)$ would render downward false alarms more likely.

While we expect the predicted anomalies to be quite general, arising in different task settings, we emphasize the moderating impact of presentation mode as a crucial boundary condition. The predicted confusion of $p$ and $n$ should be primarily, or maybe only, observed when proportions are inferred from extensional samples of elementary raw events. They would not generalize to situations in which proportions are described in normalized format, as probabilities. Thus, when the information is described as normalized probabilities (i.e., as a change from $50 \%$ to $75 \%$ ), the size of the two samples should have little impact. ${ }^{3}$

When changing proportions are neither experienced as raw observations nor described in terms of normalized probabilities but in terms of cardinal frequencies (e.g., a change from " 4 out of 8 " to " 12 out of 16 " or from " 8 out of 16 " to " 6 out of 8 "), the prediction depends on how frequencies are encoded. If they are spontaneously recoded in normalized format (e.g., as a change from $50 \%$ to $75 \%$ ) the performance should be the same as in the probability format. No anomalies should occur. If, however, cardinal frequencies are represented like enumerated samples through mental simulation of a natural sampling process (cf. Gigerenzer, 1994; Gigerenzer \& Hoffrage, 1995), then it is possible that even descriptive formats produce anomalies. Such a finding would be in line with research on the denominator neglect that also uses cardinal stimulus frequencies (cf. Reyna \& Brainerd, 2008).

Preview of experiments We first conducted several experiments with neutral, geometric stimuli to rule out any uncontrolled influence of world knowledge and affective

${ }^{3}$ Note also that, normatively, the confidence interval for the difference $\Delta=p_{\text {curr }}-p_{\text {pre }}$ is the same regardless of which one of two unequal sample sizes, $n_{\text {curr }}$ or $n_{\text {pre }}$, is larger. meaning. Experiments $1 \mathrm{a}$ and $1 \mathrm{~b}$ provide a systematic test of the predicted influences of sample size on change judgments within the experienced format only. In Experiment 2 , then, presentation mode is varied such that proportions are either experienced in cardinal-frequency format or described in probability format. The anomalies in change detection should disappear when, in the latter format, samples are presented as normalized percentages, along with the underlying $n$. The anomalies should persist when sample proportions are experienced simultaneously. This is tested in Experiment 3, in which pairs of samples are presented together and geometrical stimuli are replaced by socially meaningful smiling versus frowning faces. By manipulating the size of the left $\left(n_{\text {left }}=8\right.$ vs. 16$)$ and the right sample $\left(n_{\text {right }}=8\right.$ vs. 16$)$ orthogonally, this experiment enables us to rule out alternative accounts in terms of misunderstood task instructions or in terms of the Bayesian rule that the same large (small) proportion observed in a large sample provides stronger evidence for a large (small) $p$ than in a small sample.

\section{Experiment 1a}

\section{Method}

Participants and design Fifty-four male and female Heidelberg students participated either for payment or to meet a study requirement. In a repeated-measures design, each participant was exposed to 49 consecutive samples, calling for 48 change decisions. Two factors were varied within participants, actual changes in $p$ (large decreases, small decreases, nochange, small increases, large increases in the proportion of the focal feature) and changes in sample size (increasing from $n_{\text {pre }}=8$ to $n_{\text {curr }}=16$ vs. decreasing from $n_{\text {pre }}=16$ to $n_{\text {curr }}=8$ ).

Materials A randomized sequence of 49 stimulus samples (i.e., $48 p$-changes) was constructed under the following constraints. Within the first and last half of 24 transitions, 12 involved a change in $p$ and 12 did not. Of the 12 change trials, 3 involved large increases, 3 involved small increases, 3 small decreases, and 3 large decreases, as defined below. Large ( $n=$ 16) and small $(n=8)$ sample sizes alternated across the entire series. All levels of $p$-change appeared equally often (12 no change and 3 at each change level) with increasing and decreasing $n$. The elementary units of the binomial samples were two shaded rectangles (and from the extended ASCII set). Changes in $p$ always refer to changing proportions of the focal feature appearing in inverse video as a light rectangle against a dark background.

The algorithm used to generate the samples appears slightly complicated because it was constructed for both binary and numerical samples. Thus, rather than simply drawing $n$ stimuli 
Table 1 Means, standard deviations (parentheses), and standard errors of the mean (italics) as a function of conditions in Experiment 1a and 1b

\begin{tabular}{|c|c|c|c|c|c|c|}
\hline \multirow{2}{*}{$\begin{array}{l}\text { Change in } n \\
\text { Actual } p \text { change }\end{array}$} & \multicolumn{3}{|c|}{ Decreasing from 16 to 8} & \multicolumn{3}{|c|}{ Increasing from 8 to 16} \\
\hline & Decrease & No change & Increase & Decrease & No change & Increase \\
\hline \multicolumn{7}{|l|}{ Experiment 1a } \\
\hline Mean Judgment Score & $\begin{array}{l}-.60 \\
(.44) \\
.060\end{array}$ & $\begin{array}{l}-.28 \\
(.37) \\
.051\end{array}$ & $\begin{array}{l}-.39 \\
(.37) \\
.051\end{array}$ & $\begin{array}{l}.15 \\
(.52) \\
.071\end{array}$ & $\begin{array}{l}.24 \\
(.39) \\
.054\end{array}$ & $\begin{array}{l}.31 \\
(.42) \\
.058\end{array}$ \\
\hline Mean Accuracy Score & $\begin{array}{l}.66 \\
(.31) \\
.043\end{array}$ & $\begin{array}{l}.37 \\
(.24) \\
.033\end{array}$ & $\begin{array}{l}.14 \\
(.19) \\
.026\end{array}$ & $\begin{array}{l}.24 \\
(.25) \\
.034\end{array}$ & $\begin{array}{l}.32 \\
(.22) \\
.030\end{array}$ & $\begin{array}{l}.52 \\
(.27) \\
.037\end{array}$ \\
\hline \multicolumn{7}{|l|}{ Experiment $1 \mathrm{~b}$} \\
\hline Mean Judgment Score & $\begin{array}{l}-.33 \\
(.45) \\
.080\end{array}$ & $\begin{array}{l}-.05 \\
(.41) \\
.072\end{array}$ & $\begin{array}{l}.01 \\
(.37) \\
.062\end{array}$ & $\begin{array}{l}.05 \\
(.40) \\
.071\end{array}$ & $\begin{array}{l}.31 \\
(.36) \\
.064\end{array}$ & $\begin{array}{l}.56 \\
(.41) \\
.072\end{array}$ \\
\hline Mean Accuracy Score & $\begin{array}{l}.42 \\
(.35) \\
.062\end{array}$ & $\begin{array}{l}.46 \\
(.21) \\
.037\end{array}$ & $\begin{array}{l}.24 \\
(.20) \\
.035\end{array}$ & $\begin{array}{l}.24 \\
(.22) \\
.039\end{array}$ & $\begin{array}{l}.35 \\
.19) \\
.034\end{array}$ & $\begin{array}{l}.61 \\
(.30) \\
.053\end{array}$ \\
\hline
\end{tabular}

from a population with a binomial parameter $p$, the algorithm relies on a polygon of 49 "true" numerical values of $x$ between 0 and 1,000, which can be conceived as the expected values of the populations from which the samples are drawn. Starting from an initial value of $x=500$ on the first trial, the $x$ values on subsequent trial are generated by adding -200 (strong decrease), -100 (weak decrease), 0 (no change), +100 (weak increase) or +200 (strong increase), randomly chosen within the constraints of the aforementioned frequencies of changes in $p$ and $n$. Whenever $x$ exceeds 1,000 or falls below 0 , the sequence is discarded and the process is restarted until a full sequence remains within the 0 to 1,000 range.

For each of the 49 resulting $x_{\mathrm{i}}$ values, the algorithm then draws a random sample of size $n$ from a population with a mean of $x_{\mathrm{i}}$ and a standard deviation of 150 . The average value of this numerical sample (on the 0 -to-1,000 scale), which can be expected to deviate more from $x_{\mathrm{i}}$ when $n$ is small, is then linearly transformed to a proportional value $p$ (between 0 and 1). The number of focal features in a sample of a given size is finally chosen such that the sample proportion $P$ comes closest to the latent parameter $p$ used to generate the sample.

Procedure Groups of up to six participants worked simultaneously on separated computer workstations. All instructions and stimuli were administered on the computer screen. The full (translated) instruction text in the Appendix reveals that no cover story related the change-

${ }^{7}$ The conservative bias induced by this part of the instruction (to respond "no change" on conflicting trials) would, if anything, serve to improve the overall accuracy of no-change trials. detection task to real-world knowledge or preformed strategies. Minimal instructions simply said that the study concerned the recognition of changes in the world. Participants read that on every trial of an extended series, they would be shown a sample in which two symbols appear at different rates. They were told that the sample was drawn at random from a basic set, which determines the rate of the two symbols, and that they had to respond to the question: "Is the current sample drawn from the same basic set as the preceding sample, or has there been a change in the basic set from which the sample is drawn?" Participants were deliberately reminded of the fact that successive samples may vary even if they are drawn from the same "world," just as a consequence of statistical error. They were explicitly reminded to make inferences about changes in the world from which samples are drawn rather than merely assessing sample changes (cf. Appendix).

After two warming-up trials supposed to familiarize participants with the procedure, they were reminded once more to decide whether the current sample was drawn from the same or from a different basic set ("world") than the previous sample, emphasizing that marked fluctuations can occur even when the world has not changed and that it might therefore be wise to make a "change" response only when samples differ sufficiently. ${ }^{4}$

When participants indicated they had understood their task, they could use any keystroke to start the first trial. The 8 or 16 symbols comprising a small or large sample, respectively, were presented one by one in random locations in a $400 \times 480$ pixel black rectangular box. Stimulus elements appeared at the rate of $100 \mathrm{~ms}$ per symbol and remained on the screen. The complete sample remained 
for $3 \mathrm{~s}$ on screen and was then erased, followed by a prompt to choose between three response keys, for "Increase" $(\rightarrow)$, "Decrease" $(\longleftarrow)$, and "No-change" $(\downarrow)$ decisions. After the response, the screen was erased for $1 \mathrm{~s}$ before the next trial started. At the end of the session, participants were thanked and debriefed.

\section{Results and discussion}

Within each participant, an average change judgment score was computed for all trial types ( $p$-change $\times n$-change), coding "decrease," "unchanged," and "increase" responses as $-1,0$, and +1 , respectively. The resulting scores provided the input to two-factorial ( $p$-change $\times n$-change) repeated-measures ANOVAs. We first present the analysis of change judgments before we turn to the corresponding accuracy rates.

Change judgments To increase the reliability of these scores, judgments of strong and weak increases and of strong and weak decreases were collapsed, as averages could be based on six rather than only three data points. Inspection of Table 1 and Fig. 1 shows that the mean change-judgment scores were clearly sensitive to actual $p$-changes, as evident in the increasing judgment scores from left (decrease) to right (increase). It is also apparent, though, that change judgments were persistently higher (i.e., more positive) for increasing $n$ (dashed curve) than for decreasing $n$ (solid curve). Thus, as predicted, increasing (decreasing) sample size created a bias toward perceiving upward (downward) changes.

The resulting 3 ( $p$-change: decrease, unchanged, increase) $\times 2$ ( $n$-change) ANOVA indicates strong main effects on both repeated-measures factors. The $p$-change main effect, $F(2,106)=20.121, \eta^{2}=.275, p<.001$, reflects the sensitivity of change judgments to actual changes, evident in the upward slope of both the solid and the dashed curves in Fig. 1. However, the main effect for $n$-change was even stronger, $F(1,53)=$ 69.403, $\eta^{2}=.567, p<.001$, reflecting higher average change judgments (on the scale from -1 to +1 ) for increasing $n$ than for decreasing $n$. In Fig. 1, this is evident in the elevation of the dashed over the solid curve. The average score was higher for increasing than for decreasing $n$ in 48 of all 54 participants, suggesting a fairly regular basis for the $n$-change main effect.

A significant interaction, $F(2,106)=6.198, \eta^{2}=.105$, $p=.003$, is mainly due to a reduced sample-size effect for no-change trials (cf. middle points of the two curves). Nevertheless, no-change trials solicited clearly more "increase" judgments when $n$ increased $(M=+.24)$; $t(53)=4.57, d=1.255, p<.001$, but more "decrease" judgments when $n$ decreased $(M=-.28) ; t(53)=-5.54$, $d=1.522, p<.001$.

\section{Mean Change-Judgment Score}
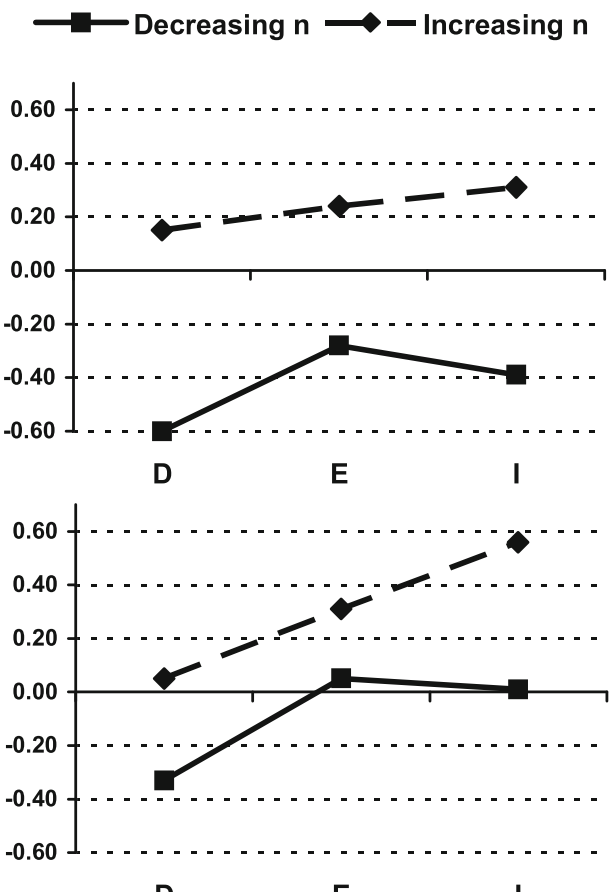

D
E

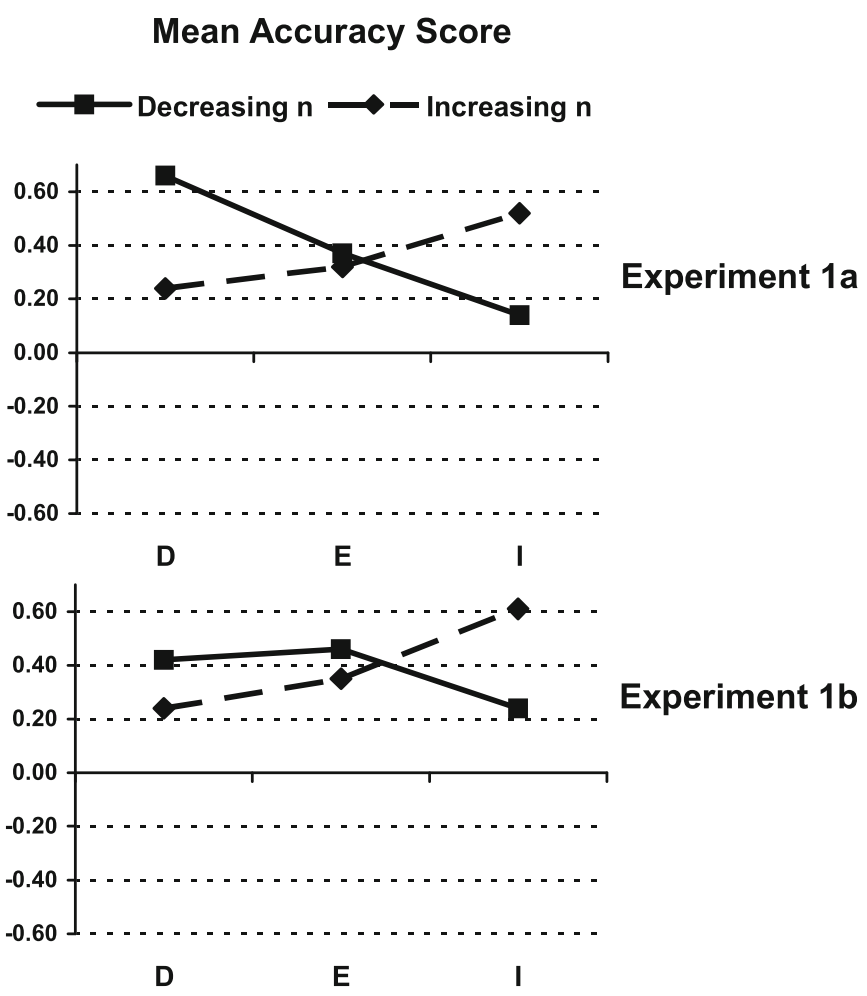

D
$\mathbf{E}$

Fig. 1 Mean change judgment and mean accuracy scores in Experiment 1a (upper part) and Experiment $1 \mathrm{~b}$ (lower part) as a function of experimental conditions: decreasing vs. increasing sample size on decreasing (D), equal (E), and increasing (I) trials 
Accuracy As a consequence of the impact of $n$-changes on the detection of latent $p$-changes in the environment, there are corresponding differences in accuracy. Correct responses (i.e., "decrease," "no-change," or "increase" judgments on decrease, no-change, or increase trials, respectively) were coded +1 ; incorrect responses were coded 0 . Thus, the average accuracy score corresponds to the proportion of correct responses across all trials. The right chart of Fig. 1 reveals that correctness was regularly enhanced when changes in $n$ were in the same direction as changes in $p$. For decreasing $n$, the mean accuracy scores declined from downward-change $(M=.66)$ to no-change $(M=.37)$ to upward-change trials $(M=.14)$. The opposite was the case for increasing $n(M=.24$ vs. .32 vs. .52$)$. In the accuracy ANOVA, these reverse trends gave rise to a strong interaction effect, $F(2,106)=73.625$, $\eta^{2}=.581, p<.001$, as manifested in the crossover in the upper right chart in Fig. 1. The interaction was slightly asymmetric; somewhat higher accuracies for decreases than for increases produced a change-direction main effect, $F(2,53)=4.737, \eta^{2}=.082, p=.0128$.

\section{Correspondence of judgments with given sample Note in} passing that similar results were obtained for the consistency of judgments with actually observed sample changes, as distinguished from accurate population inferences. Again, an ANOVA of the proportions of change judgments consistent with the direction of change between the current and the sample only resulted in a significant $p$-change $\times n$-change interaction, $F(2,106)=22.463, \eta^{2}=.298, p<.001$. The impact of changing $n$ on sample consistency was somewhat less pronounced, though, than on accurate inferences. When $n$ decreased, consistency scores decreased from .491 to .451 to .401 for decreasing, equal, and increasing transitions. When $n$ increased, consistency increased from .380 to .401 to .556 . For comparison, the corresponding decreases and increases in the accuracy of population inferences amount to $.66, .37, .14$ and $.24, .32, .52$, respectively (see Table 1 ). Thus, the impact of $n$-changes on the detection of $p$-changes seems to affect population inferences more than the assessment of manifest sample proportions.

Altogether, these results support the theoretical predictions in virtually all respects. First of all, change judgments were generally accurate and sensitive to the sign and size of actually presented changes. However, despite this basic sensitivity of change judgments, the predicted anomalies were borne out at high effect sizes. On no-change trials, increasing sample size led to many false increase judgments whereas decreasing sample size led to many false decrease judgments. Actual downward changes were more readily detected when $n$ decreased than when $n$ increased. Conversely, the detection of upward changes profited from an increasing $n$. The general bias to respond "decrease" when $n$ decreased but "increase" when $n$ increased led to enhanced accuracy when changes in $n$ were congruent with changes in the critical proportion $p$, but to reduced accuracy for incongruent transitions.

Thus, the first set of predictions about the moderating impact of sample size on change detection was clearly supported. However, before we move on to testing the second set of predictions regarding the impact of presentation modes, we consider a replication experiment that was run to rule out a superficial alternative account. As the focus was always on the proportion of the lighter one of two rectangles, it is possible that the strength of findings is, to an unknown degree, peculiar to the perceptual salience of the focal feature. We therefore ran a comparison experiment in which the seemingly less salient rectangle was the focal feature.

\section{Experiment 1b}

\section{Method}

Thirty-two male and female students from the same undergraduate pool participated in this experiment, which followed the same design and the same experimental task as in Experiment 1a, with only one exception. Participants were instructed to detect changes in the proportion of the dotted rectangle that contrasted less with the black background color of the screen than the filled rectangle - This was accomplished by simply exchanging the roles played by the two binary features in the instructions and in the stimulus materials.

\section{Results and discussion}

A two-factorial $p$-change $\times n$-change ANOVA yielded the same pattern of significant findings as in the first experiment (cf. Table 1), as apparent from a comparison of the upper and lower part of Fig. 1. Again, a main effect for $p$-changes, $F(2$, $62)=82.409, \eta^{2}=.727, p<.001$, testifies to the basic accuracy of change judgments. However, a main effect for $n$-changes, $F(1,31)=10.493, \eta^{2}=.253, p=.003$, again reflects higher change judgments for increasing than decreasing $n$. This inequality (of average change judgments for decreasing and increasing $n$ ) held for 23 of all 32 judges. The significant interaction, $F(2,62)=11.183, \eta^{2}=.265, p<.001$, is again related to a reduced sample-size effect for no-change trials.

Consistent with the first experiment, the major result in the ANOVA of the accuracy scores was again a cross-over interaction (see right chart in Fig. 1), $F(2,62)=14.849, \eta^{2}=.324$, $p<.001$. Accuracy increased when $n$-changes matched $p$ changes, that is, when increases in $p$ came along with increases in $n$ and when decreases in $p$ came along with decreases in $n$. In contrast, accuracy was low when there was a mismatch between $p$-changes and $n$-changes. The main 
effects, for $p$-changes, $F(2,62)=2.312, p=.108$, and for $n$ changes, $F(1,31)=1.602, p=.215$, were not significant.

Thus, whether the focus was on the lighter or the darker feature, a similar pattern emerged. Basically, sensitive judgments were biased by the sample size manipulation. An increasing $n$ facilitated the detection of increases and impaired the detection of decreases in $p$. A decreasing $n$ caused a similar bias to detect decreases but to fail detecting increases.

To directly test sensitivity to proportional change, beyond the minimalist strategy to assess changes in the number of focal features, we regressed each participant's 48 change judgments on two predictors, the proportional change in $p$ and the change in the frequency of the focal feature. Pooling over all 86 participants from Experiments $1 \mathrm{a}$ and $1 \mathrm{~b}$, the regression weights of both predictors were clearly positive. Change judgments were not only affected by changes in the frequency of the focal feature, mean $\beta=+.37, S D=.37, t(85)=9.279, d=$ 2.013, $p<.001$. Rather, the actual $p$-changes had a similarly strong impact, mean $\beta=+.21, S D=24, t(85)=8.129, d=1.763$, $p<.001$. The average correlation (redundancy) between predictors was only $r=.30(S D=.17)$. This apparent sensitivity to $p$, when $n$ is controlled for, indicates that judges did not simply misunderstand the task instructions and merely attend to changes in the frequency of the focal feature. They were actually sensitive to changes in $p$.

\section{Experiment 2}

The strength of the anomalies resulting from the confusion of $n$ and $p$ is notable. Actual decreases (increases) in $p$ were often completely overridden by increases (decreases) in $n$, and $n$ changes on no-change trials induced strong false alarms. Such anomalies in the detection of change have apparent practical and theoretical implications. The number of observations can greatly obscure the perceived changes in opinions, market indices, or student performance.

The experienced presentation mode that was used in Experiments $1 \mathrm{a}$ and $1 \mathrm{~b}$ appears to be particularly prone to producing strong anomalies. As already noted, when $p$ has to be learned inductively from an experienced sample, the impact of singular elements or subsets of a growing sample on the continuous update of $p$ is hard to determine. In contrast, describing a sample proportion in a normalized probability format should greatly facilitate the encoding of $p$ and of $n$. Thus, $75 \%$ in a small sample $(n=8)$ is easily recognized as a percentage equivalent to $75 \%$ in a large sample $(n=16)$, even though $n$ is given explicitly.

For an empirical test of these considerations, we manipulated the presentation mode in Experiment 2. The strong impact of $n$ on the assessment of $p$ should be replicated once more in an experienced-format condition. However, the anomalies should be markedly reduced, or even fully eliminated, in a descriptive probability-plus- $n$ condition, which provided sample percentages along with the relevant $n$, and the same prediction holds, of course, when $n$ is not provided in a probability-only baseline condition. Note that although the latter condition seems to render any impact of $n$ on $p$ trivially impossible, it affords a natural baseline or control condition for the accuracy of change inferences when sample proportions are described as normalized percentages, in the absence of $n$ information. Convergent results obtained in both percentage conditions, with and without $n$, would highlight that sample size is practically ignored in the percentage format condition.

Yet, even with a descriptive format, predictions may be less clear-cut when proportions are described as cardinal frequencies rather than normalized probabilities. Although " 12 out of 16 " conveys similar information as " $75 \%$ at sample size 16 ," the two formats may not be equivalent psychologically (Fiedler, 1988; Gigerenzer, 1991, 1994). Natural frequencies may encourage an enumerative induction process, whereby the experience of 12 out of 16 elements might be mentally simulated, leading to similar results as an experienced format.

\section{Method}

Participants and design One hundred and twenty-six male and female students of the University of Heidelberg participated. They were divided into four experimental groups receiving different presentation formats. ${ }^{5}$ The experiencedmode group constitutes a replication of Experiment 1a. The other three groups were assigned different descriptive formats: a probability-plus- $n$ format, a probability-only baseline format without $n$, and a cardinal-frequency format.

Materials and procedures The same task instructions and the same computer algorithm as in Experiment 1a were used to generate sequences of 49 of $p$ and $n$-changes. Again, the first and second half of the stimulus series consisted of 24 transitions each, including 12 no-change trials, 6 upward and 6 downward changes, half of which were weak and strong. The procedure was only modified in the descriptive-format conditions: In the probabilityplus- $n$ condition, the percentages of the focal and the nonfocal features appeared as two lines of enlarged text within the same rectangular box as the experienced samples. For instance, participants read, in four lines of centered text: "Drawn were / 8 (16) symbols / 75

${ }^{5}$ The allocation of participants to conditions was only quasirandom, as the number of participants in the two probability conditions was finally increased to allow for a more reliable check on the assumption that sample/size effects are absent in these conditions. 
Percent / 25 Percent " (where "/" denotes line breaks). For the probability-only baseline condition, the same display was used except that the sample-size line was omitted. In the cardinal-frequency format condition participants were presented with equivalent quantities expressed as natural frequencies, for example, "12 $\square /$ 4 . ." Note that the sum of both natural frequencies also reveals the size of the sample.

\section{Results and discussion}

Within participants, mean change-judgment scores (coding "decrease," "unchanged," and "increase" responses as $-1,0$, and +1 , respectively) and mean accuracy scores were computed for all combinations of $p$-change (decrease, equal, increase) and $n$-change (decreasing $n$ vs. increasing $n$ ). For ANOVA purposes, again, all decreasing and increasing trials were pooled. Table 2 provides average scores and standard deviations by experimental conditions. Graphical illustrations of the same results appear in Fig. 2.
Experienced format All characteristic results were replicated in the experienced-format condition (see upper left chart of Fig. 2). A main effect for actual $p$-changes, $F(2$, $42)=41.79, \eta^{2}=.666, p<.001$, again accompanied by a main effect for $n$-changes, $F(1,21)=8.48, \eta^{2}=.288$, $p=.008$, reflects both a basic sensitivity to $p$-changes but also a bias toward higher "increase" judgments for increasing than for decreasing $n$. In this study, though, $n$ changes did not equally affect "decrease" judgments (as evident from the asymmetric pattern in Fig. 2), yielding a significant interaction, $F(2,42)=32.76, \eta^{2}=.609, p<.001$.

In the accuracy ANOVA, too, the interaction, $F(2,42)=$ $7.80, \eta^{2}=.271, p=.001$, is due to increasing $n$ enhancing accurate detection of actual increases. As decreasing $n$ did not similarly facilitate detection of decreases, the asymmetry emerges as a main effect for $n$-changes, $F(1,21)=45.17$, $\eta^{2}=.683, p<.001$. Increasing $n$ led to generally higher accuracy. We do not have any explanation for this divergence of a single data point. In any case, however, both main effects were replicated once more, corroborating the contention that

Table 2 Means, standard deviations (parentheses), and standard error (italics) in Experiment 2

\begin{tabular}{|c|c|c|c|c|c|c|}
\hline \multirow{2}{*}{$\begin{array}{l}\text { Change in } n \\
\text { Actual } p \text { change }\end{array}$} & \multicolumn{3}{|c|}{ Decreasing from 16 to 8} & \multicolumn{3}{|c|}{ Increasing from 8 to 16} \\
\hline & Decrease & No change & Increase & Decrease & No change & Increase \\
\hline \multicolumn{7}{|l|}{ Experienced Mode } \\
\hline Mean Judgment Score & $\begin{array}{l}-.23 \\
(.43) \\
.092\end{array}$ & $\begin{array}{l}-.18 \\
(.34) \\
.072\end{array}$ & $\begin{array}{l}.05 \\
(.38) \\
.081\end{array}$ & $\begin{array}{l}-.27 \\
(.35) \\
.075\end{array}$ & $\begin{array}{l}.36 \\
(.35) \\
.075\end{array}$ & $\begin{array}{l}.56 \\
(.31) \\
.066\end{array}$ \\
\hline Mean Accuracy Score & $\begin{array}{l}.38 \\
(.30) \\
.064\end{array}$ & $\begin{array}{l}.43 \\
(.31) \\
.066\end{array}$ & $\begin{array}{l}.27 \\
(.25) \\
.053\end{array}$ & $\begin{array}{l}.44 \\
(.22) \\
.047\end{array}$ & $\begin{array}{l}.42 \\
(.28) \\
.060\end{array}$ & $\begin{array}{l}.66 \\
(.25) \\
.053\end{array}$ \\
\hline \multicolumn{7}{|l|}{$\begin{array}{l}\text { Descriptive Mode } \\
\quad \text { (Cardinal Frequencies) }\end{array}$} \\
\hline Mean Judgment Score & $\begin{array}{l}-.21 \\
(.29) \\
.062\end{array}$ & $\begin{array}{l}-.06 \\
(.31) \\
.066\end{array}$ & $\begin{array}{l}.34 \\
(.35) \\
.075\end{array}$ & $\begin{array}{l}.08 \\
(.28) \\
.060\end{array}$ & $\begin{array}{l}.33 \\
(.26) \\
.055\end{array}$ & $\begin{array}{l}.57 \\
(.32) \\
.068\end{array}$ \\
\hline Mean Accuracy Score & $\begin{array}{l}.23 \\
(.28) \\
.060\end{array}$ & $\begin{array}{l}.61 \\
(.26) \\
.055\end{array}$ & $\begin{array}{l}.42 \\
(.28) \\
.060\end{array}$ & $\begin{array}{l}.20 \\
(.11) \\
.023\end{array}$ & $\begin{array}{l}.48 \\
(.29) \\
.062\end{array}$ & $\begin{array}{l}.61 \\
(.29) \\
.062\end{array}$ \\
\hline \multicolumn{7}{|l|}{$\begin{array}{l}\text { Descriptive Mode } \\
\quad \text { (Probability-plus- } n \text { ) }\end{array}$} \\
\hline Mean Judgment Score & $\begin{array}{l}-.13 \\
(.28) \\
.050\end{array}$ & $\begin{array}{l}.02 \\
(.22) \\
.040\end{array}$ & $\begin{array}{l}.23 \\
(.28) \\
.050\end{array}$ & $\begin{array}{l}-.19 \\
(.26) \\
.047\end{array}$ & $\begin{array}{l}-.08 \\
(.25) \\
.045\end{array}$ & $\begin{array}{l}.11 \\
(.20) \\
.036\end{array}$ \\
\hline Mean Accuracy Score & $\begin{array}{l}.32 \\
(.23) \\
.041\end{array}$ & $\begin{array}{l}.58 \\
(.24) \\
.043\end{array}$ & $\begin{array}{l}.31 \\
(.17) \\
.031\end{array}$ & $\begin{array}{l}.33 \\
(.17) \\
.031\end{array}$ & $\begin{array}{l}.54 \\
(.28) \\
.050\end{array}$ & $\begin{array}{l}.28 \\
(.19) \\
.034\end{array}$ \\
\hline \multicolumn{7}{|l|}{ Probability only } \\
\hline Mean Judgment Score & $\begin{array}{l}-.24 \\
(.27) \\
.038\end{array}$ & $\begin{array}{l}-.01 \\
(.17) \\
.024\end{array}$ & $\begin{array}{l}.18 \\
(.33) \\
.046\end{array}$ & $\begin{array}{l}-.22 \\
(.26) \\
.036\end{array}$ & $\begin{array}{l}-.06 \\
(.16) \\
.022\end{array}$ & $\begin{array}{l}.20 \\
(.33) \\
.046\end{array}$ \\
\hline Mean Accuracy Score & $\begin{array}{l}.39 \\
(.21) \\
.029\end{array}$ & $\begin{array}{l}.62 \\
(.16) \\
.022\end{array}$ & $\begin{array}{l}.32 \\
(.23) \\
.032\end{array}$ & $\begin{array}{l}.35 \\
(.20) \\
.028\end{array}$ & $\begin{array}{l}.56 \\
(.18) \\
.025\end{array}$ & $\begin{array}{l}.27 \\
(.29) \\
.041\end{array}$ \\
\hline
\end{tabular}


change judgments are sensitive to real $p$-changes as well as irrelevant changes in $n$.

Probability-plus-n format In the ANOVA of change judgments in the probability-plus- $n$ condition, the confusion of $p$ and $n$-changes was completely eliminated. A strong $p$ change main effect, $F(2,60)=43.64, \eta^{2}=.593, p<.001$, reflecting high sensitivity to normalized quantities, was no longer accompanied by a main effect for $n$-changes, $F(1,30)=3.857, \eta^{2}=.114, p=.059$. If anything, the nonsignificant difference points in the opposite direction (cf. Fig. 2). Judgment scores were slightly higher for decreasing $n$ (solid line) than for increasing $n$ (dashed line). The interaction was negligible, $F<1$.

Again, the inverted-V curve for accuracy scores (on right side of Fig. 2) reflects the higher accuracy of no-
Mean Change-Judgment Score

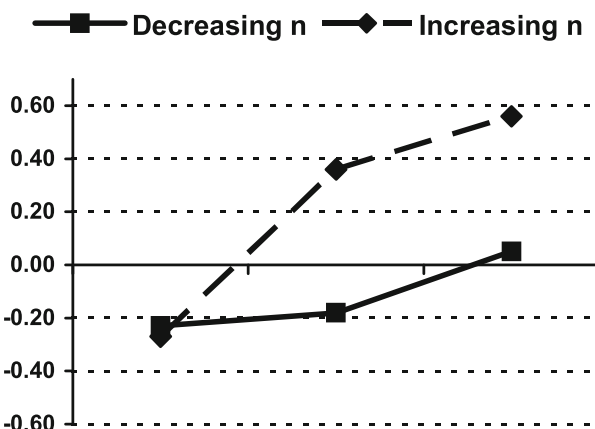

D

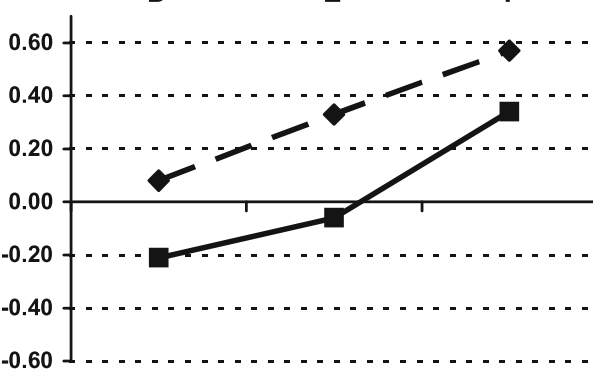

D

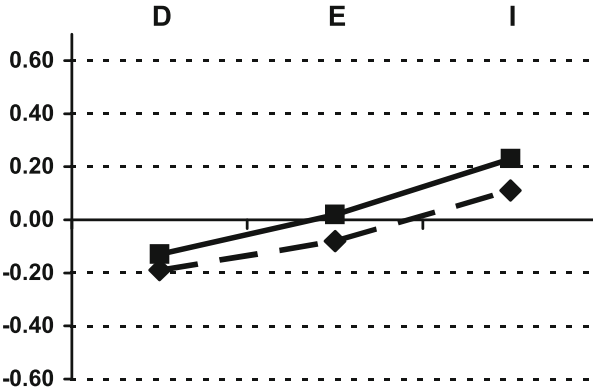

D

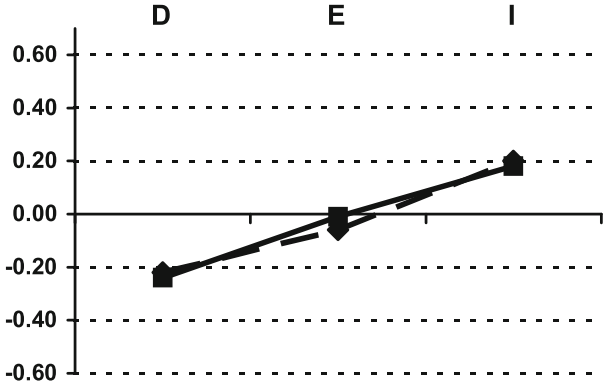

D

E

I

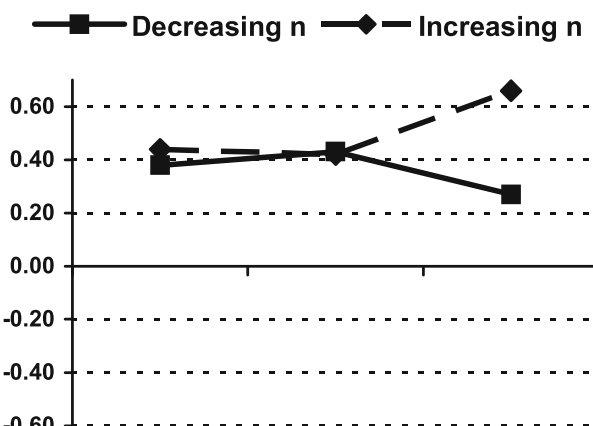

D

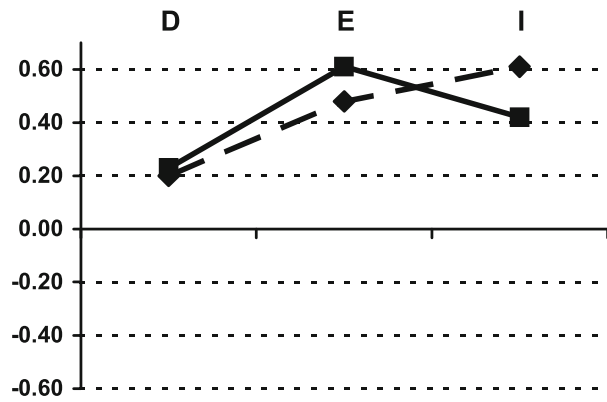

D

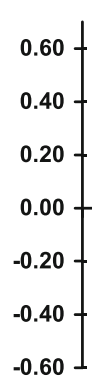

..

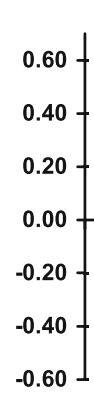

Mean Accuracy Score

\section{Experienced} Mode

\section{Cardinal \\ Frequency \\ Format}

\section{Probability plus $n$ Format}

\section{Probability \\ only Format \\ (Baseline)}

Fig. 2 Mean change judgment and mean accuracy scores for different presentation format conditions of Experiment 2, as a function of experimental conditions: decreasing vs. increasing sample size on decreasing (D), equal (E), and increasing (I) trials 
change than change decisions, which is due to a response bias toward "no-change" responses (cf. Footnote $4)$. The corresponding $p$-change main effect amounts to $F(2,60)=14.611, \eta^{2}=.328, p<.001$. No other $F$ test was significant, $F_{\mathbf{s}}<1$.

Probability-only format Virtually the same pattern was obtained in this baseline condition, in which probabilities were provided without sample-size information. The only significant result in the ANOVA of change-judgment scores was a strong main effect for $p$-change, $F(2$, 100) $=63.94, \eta^{2}=.561, \mathrm{p}<.001$ (other $F \mathrm{~s}<1$ ). The almost perfect overlap of both graphs and the resemblance of the graphs for this and previous conditions together strongly suggest that sample size was simply ignored in probability-format conditions (cf. Fig. 2). Moreover, the symmetry of the two curves around zero suggests that, given normalized probabilities, actual increases and decreases were detected about equally well.

The accuracy ANOVA only revealed a main effect for $p$ changes, $F(2,100)=42.77, \eta^{2}=.461, p<.001$, reflecting the same inverted $\mathrm{V}$ as in all descriptive conditions (see lower right part of Fig. 2). No changes led to more accurate decisions than changes (cf. Footnote 4). A slight tendency for decreasing $n$ to produce more accurate judgments than increasing $n$ was not significant, $F(1,50)=2.739, p=.104$. The interaction was negligible, $F<1$.

Cardinal-frequency format Would a descriptive cardinalfrequency format yield similar results as the descriptive percentage formats, or would cardinal frequencies produce the same anomalies as the experienced-format condition? A glance at Fig. 2 shows that the results support the latter option. Describing the frequency of focal and nonfocal features yielded similar judgment biases as the inductive experience of a feature set presented graphically on the screen. In the ANOVA of change judgment scores, this was again evident in main effects for actual $p$-changes, $F(2,42)=64.91, \eta^{2}=.756, p<.001$, and $n$ changes, $F(1,21)=19.65, \eta^{2}=.483, p<.001$. No interaction was found, $F(2,42)=.423$.

In the accuracy analysis, this led to a significant $p$ change $\times n$-change interaction, $F(2,42)=5.01, \eta^{2}=.193$, $p=.011$, reflecting the typical pattern obtained with experienced formats. Accuracy in detecting increases was enhanced when $n$ increased, whereas accuracy in detecting decreases profited from decreasing $n$ (see right side of Fig. 2). The performance on no-change trials is complicated by an apparent bias to guess "unchanged" rather than "changed." This led to enhanced accuracy for no-change trials, causing a $p$-change main effect $F(2$, $42)=12.06, \eta^{2}=.365, p<.001$. The $n$-change main effect was negligible, $F(1,21)=0.05$.
Comparisons between format conditions Planned contrasts corroborate the contention that the experienced and the cardinal-frequency mode produced similarly strong anomalies that were eliminated in the probability-plus- $n$ condition and in the probability-only condition. For a convenient index of the sample-size effect, we computed within each participant the average difference $D_{\mathrm{n}}$ between change judgments for all increasing- $n$ minus all decreasing- $n$ trials. This score reflects the strength of the $n$-change main effect in individual judges.

Consistent with the ANOVA results, no significantly positive difference score was obtained for the probabilityonly condition, mean $D_{\mathrm{n}}=-0.005, S D=0.159, t(50)=$ $0.221, d=0.063, p=.826$, and the probability-plus- $n$ condition, mean $D_{\mathrm{n}}=-0.089, S D=0.251, t(30)=-1.964, d=0.717$, $p=.059$. Both conditions did not differ significantly, $t(80)=-1.853, d=0.414, p=.068$. If anything, the negative $D_{\mathrm{n}}$ in the latter condition is opposite to the positive $n$ change effect in the experienced mode, $D_{\mathrm{n}}=+1.015, S D=$ $1.635, t(21)=+2.912, d=1.271, p=.008$, and in the cardinalfrequency condition, $D_{\mathrm{n}}=+0.920, S D=0.974, t(21)=+4.432$, $d=1.934, p<.001$. Both of these conditions differed significantly from the probability conditions, $t(51)=+3.836, d=$ $1.074, p<.001$, (experienced) and $t(51)=+4.997, d=1.399$, $p<.001$, (cardinal frequency), but not from each other, $t(42)=+0.233, d=0.072, p=.817$.

Thus, the detection of changes in $p$ was contingent on congruent changes in $n$ when samples were experienced and also when proportions were described as cardinal frequencies. As expected, however, the anomalies disappeared when proportions were described as normalized probabilities. It did not matter whether $n$ was provided or not, suggesting that normalized probabilities render change detection insensitive to the provision of $n$.

\section{Experiment 3}

Despite the moderating impact of presentation format, the intrusion of $n$ into the assessment of $p$ turns out to represent a general and robust phenomenon. To highlight its generality, we conducted another experiment, in which meaningless geometric symbols were replaced by smiling versus frowning faces and a meaningful cover story was introduced, inviting participants to take the role of a politician who is to detect differences in the rate of positive facial expressions in different audiences. Moreover, by presenting pairs of samples simultaneously on the left and right half of the screen rather than successively on subsequent screens, Experiment 3 allowed us to study if the phenomenon extends to spatial changes or differences, from left to right, beyond merely temporal changes in time. Note that within this simultaneous version of the task, the same predictions can be 
applied in both directions. When $n_{\text {left }}$ is smaller than $n_{\text {right }}$, this should facilitate detecting increases from left to right or else decreases from right to left, and vice versa. For convenience, we continue to refer to detection of "changes," although sample proportions now vary in space rather than in time. In either case, the task calls for comparing two proportions, only one of which can be focused at a time.

However, apart from the generality of the phenomenon, another purpose of Experiment 3 was to provide further evidence for the foremost premise that the change detection task was understood correctly. That is, we wanted to rule out the possibility that in spite of the explicit instructions (see Appendix) judges may have not understood that they were to assess $p$-changes independently of $n$-changes but may have exclusively assessed changes in the frequency of the focal feature. That this was not the case was already strongly suggested by the results of regression analyses (conducted within individual participants), showing that change judgments were regularly affected by $p$-changes independently of $n$-changes. However, for a more cogent test of the premise that judgments were sensitive to changes in $p$ and not just to differences in the absolute frequency of the focal feature, it would be necessary to include trials that provide $p$-changes when $n$ remains constantly high $(n=16)$ or low $(n=8)$. If participants merely assess changes in the number of the focal feature, then the same $p$-changes should appear stronger for a constant $n=16$ than for $n=8$. For instance, a decrease from 12 out of 16 to 8 out of 16 in a large sample entails a stronger decrease in the focal feature rate (from 12 to 8 ) and should thus lead to stronger change judgments than a decline from 6 (out of 8 ) to 4 (out of 8) in a small sample.

Experiment 3 affords a systematic test of these considerations because the size of the left and right sample was manipulated orthogonally, yielding all combinations of unequal $\left(n_{\text {left }}=8, n_{\text {right }}=16\right.$ or $n_{\text {left }}=16, n_{\text {right }}=$ 8 ) and equal $\left(n_{\text {left }}=8, n_{\text {right }}=8\right.$ or $\left.n_{\text {left }}=16, n_{\text {right }}=16\right)$ samples sizes. If the detection of the same $p$-changes on equal- $n$ trials (e.g., from $12 / 16=6 / 8=p=.75$ to $8 / 16=4 / 8=p=.5$ ) is not affected by sample size, although the same anomalies as in the other experiments are obtained on unequal- $n$ trials, this would be incompatible with the notion that judges were exclusively assessing frequencies rather than proportions.

\section{Method}

Participants and design Fifty-two male and female students at the University of Heidelberg participated either for payment (4 Euro) or to meet a course requirement.
Three factors were manipulated within participants across 64 trials. Every trial involved the comparison of two samples, presented on the left and right side of the screen. The change in the proportion of smiling faces (from left to right) was manipulated in the population from which the sample pairs were drawn. To increase the external validity, a wider range of $p$ differences was covered. That is, $p$ differences were set to 0 on 16 trials, and to increases and decreases of .2, .4 , and .6 on 12,8 , and 4 trials, respectively. One fourth of the trials at each level of $p$-change was paired with each of the four $n$ combinations (i.e., $n_{\text {left }}=8$ vs. 16 along with $n_{\text {right }}=8$ vs. 16 ).

Materials and procedure All instructions, stimulus samples, and dependent measures were controlled by a Visualbasic.net program. The initial instructions mentioned that the ability to quickly capture approval or rejection in one's social environment constitutes a basic module of social intelligence. Participants read the following:

Please take the role of a politician who, in an election campaign, is giving many public speeches and who has to infer the degree of agreement versus disagreement from the faces in the audience. More specifically, you should find out on each trial which one of two political proposals found more agreement. You will be presented on each side of the screen two snapshots of reactions to two proposals, and you should decide, if one of them found more agreement.

To emphasize the distinction between a sample and the underlying reality, participants then read on a new screen:

For this purpose, it is not only important to grasp several people's reactions at once. You also have to consider that every trial provides only a snapshot of a few people. Your task as a politician, however, is to decide whether there is more agreement for one of the two proposals in the population. An essential aspect of social intelligence, which is important here, is this generalizing inference.

Each trial consisted of two samples of 8 or 16 faces generated by the FaceGen software (http://www.facegen.com). Samples were randomly drawn from a basic set of 32 adult White faces (16 female and 16 male), for which a positive expression (smile open 1.0, smile closed 1.0) and a negative impression (FaceGen parameter disgust 1.0) was available. The binomial probability of presenting the positive expression of each of the $n$ faces was determined by the parameter $p$. The two sets of $n_{\text {left }}$ and $n_{\text {right }}$ (size: width 60 pixels, height 87 pixels) faces were presented 
simultaneously in random locations of two windows covering the left and right side of the screen. After a presentation time of 2,500 ms, too short to allow for counting, the screen was erased and a decision was prompted between "More acceptance on the left," "More acceptance on the right," and "Relatively equal." Participants were asked to click on one option and then to indicate their subjective confidence on a 5 -point scale, ranging from very unconfident to very confident. Finally, participants were thanked, debriefed, and paid.

\section{Results and discussion}

Dependent measures were subjected to repeated-measures ANOVAs with three factors, $p$-change (decreasing vs. equal vs. increasing), $n_{\text {left }}(8$ vs. 16$)$, and $n_{\text {right }}(8$ vs. 16$)$.

Change judgments Average change judgments (coding 1,0 , and +1 for "decrease," "no change," and "increase, " respectively) appear in Fig. 3 and in Table 3 as a function of experimental conditions. As in all previous experiments, comparisons of simultaneously experienced samples were not only sensitive to true changes from $p_{\text {left }}$ to $p_{\text {right }}$ but also to the relation of $n_{\text {left }}$ and $n_{\text {right }}$. The strong upward slope of all curves in Fig. 3 shows that change judgments increased monotonically from decreasing $-p$ to equal- $p$ to increasing $-p$ trials, reflecting basic $p$ sensitivity. However, the vertical difference between the two curves on the left, which only refer to unequal- $n$ trials, also indicates that increasing $n$ (dashed line) led to higher change judgments than decreasing $n$ (solid line). In contrast, when $n_{\text {left }}$ and $n_{\text {right }}$ did not differ, judgments also reflected actual $p$-changes accurately but sample size did not matter at all, as evident from the almost perfect congruence of both lines in the

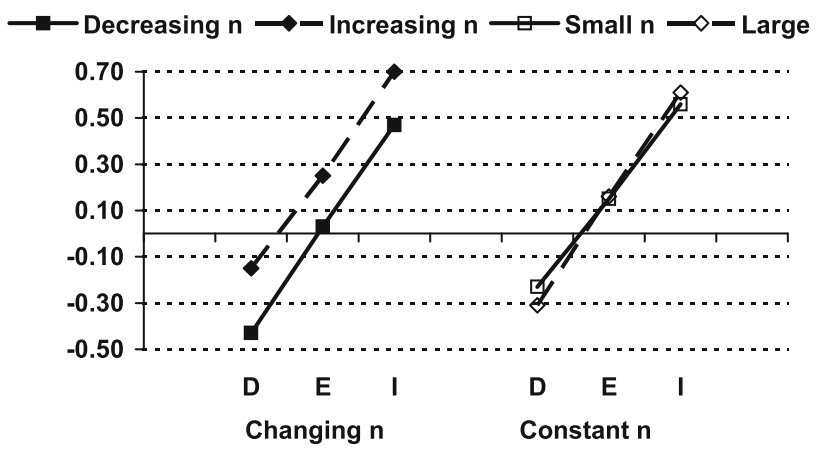

Fig. 3 Mean change judgments in Experiment 3 as a function of experimental conditions. As evident from the upward slope of all curves, change judgments clearly distinguished between decreasing (D), equal (E), and increasing (I) proportions. The vertical displacement of the two curves on the left reflect that increasing sample size $n$ (dashed curve) led to higher change judgments than decreasing $n$ (solid curve). However, the almost perfectly congruent curves on the right show that constantly small (solid) versus large (dashed) sample size led to similar change judgments right part of Fig. 3. Thus, the same changes in $p$ did not lead to stronger change judgments when $n$ was doubled, even though the frequency difference is also doubled when sample size increases from 8 to 16 . Thus, the absence of any influence of sample-size on equal $n$ trials is incompatible with a misunderstanding of the task instructions.

Note also that the dashed curve on the left (for $n_{\text {left }}=8$ increasing to $n_{\text {right }}=16$ ) is elevated relative to the dashed curve on the right (for $n_{\text {left }}=n_{\text {right }}=16$ ), reflecting higher change judgments when $n_{\text {left }}$ is reduced. Similarly, the solid curve on the left (for $n_{\text {left }}=16$ decreasing to $n_{\text {right }}=8$ ) is lower than the solid curve on the right (for $n_{\text {left }}=n_{\text {right }}=8$ ), reflecting higher change judgments when $n_{\text {right }}$ increases.

A three-factorial ANOVA corroborates these descriptive findings. Marked main effects for $n_{\text {left }}, F(1,51)=25.917$, $\eta^{2}=.337, p<.001$, and for $n_{\text {right }}, F(1,51)=13.565$, $\eta^{2}=.191, p=.001$, were obtained along with the overwhelming main effect for actual $p$-changes, $F(2,102)=$ $184.535, \eta^{2}=.783, p<.001$. Two-way and three-way interactions were negligible, $F_{\mathbf{S}}<2$.

The inclusion of a confidence measure in Experiment 3 provided an opportunity to run an ANOVA with a confidence-weighted judgment score, defined as the product of +1 and -1 (for "increase" and "decrease") with confidence ratings on the scale from 1 to 5 . The same pattern was obtained: a change-level main effect, $F(2,102)=27.258, \eta^{2}=.348$, $p<.001, p$-change interactions with $n_{\text {left }}, F(1,51)=13.018$, $\eta^{2}=.203, p<.001$, and $n_{\text {right }}, F(1,51)=8.378, \eta^{2}=.141$, $p=.006$, and a three-way interaction, $F(2,102)=9.027$, $\eta^{2}=.150, p<.001$.

Thus, when $n_{\text {left }}$ and $n_{\text {right }}$ vary orthogonally, both samples sizes contribute to the impact of changing samples size. At the same time, the invariant slope for constantly small and large samples in Fig. 3 suggests, once more, that change judgments are first of all sensitive to proportions, and anomalies only arise when there is a (misleading) change in sample size. The complete elimination of sample-size effects on equal- $n$ trials is inconsistent with the notion that judgments reflect focal-feature differences rather than proportions.

Accuracy A corresponding pattern arises again in the analysis of decision accuracy scores. Crucially, the impact of sample size shows up in two interactions, $p$ change $\times n_{\text {left }}, F(1,51)=10.651, \eta^{2}=.173, p=.002$, and $p$-change $\times n_{\text {right }}, F(1,51)=5.596, \eta^{2}=.099, p=.022$. Detecting decreases is facilitated when $n_{\text {left }}$ is large and

${ }^{\circ}$ Change assessment in this experiment was inferred from the sensitivity of repeated teachers' judgments to actually occurring changes in student performance rather than direct judgments of change. 
Table 3 Means, standard deviations (parentheses), and standard errors of the mean (italics) as a function of conditions in Experiment 3

\begin{tabular}{|c|c|c|c|c|c|c|}
\hline \multirow{2}{*}{$\begin{array}{l}\text { Changing } n \\
\text { Actual } p \text { change }\end{array}$} & \multicolumn{3}{|c|}{ Decreasing from 16 to 8} & \multicolumn{3}{|c|}{ Increasing from 8 to 16} \\
\hline & Decrease & No change & Increase & Decrease & No change & Increase \\
\hline Mean Judgment Score & $\begin{array}{l}-.43 \\
(.61) \\
.085\end{array}$ & $\begin{array}{l}0.03 \\
(.50) \\
.069\end{array}$ & $\begin{array}{l}0.47 \\
(.38) \\
.053\end{array}$ & $\begin{array}{l}-.15 \\
(.54) \\
.075\end{array}$ & $\begin{array}{l}.25 \\
(.50) \\
.069\end{array}$ & $\begin{array}{l}.70 \\
(.24) \\
.033\end{array}$ \\
\hline Constant $n$ & $n=8$ & & & $n=16$ & & \\
\hline Mean Judgment Score & $\begin{array}{l}-.23 \\
(.55) \\
.076\end{array}$ & $\begin{array}{l}.15 \\
(.46) \\
.064\end{array}$ & $\begin{array}{l}.56 \\
(.33) \\
.046\end{array}$ & $\begin{array}{l}-.31 \\
(.58) \\
.080\end{array}$ & $\begin{array}{l}.16 \\
(.49) \\
.082\end{array}$ & $\begin{array}{l}.61 \\
(.27) \\
.037\end{array}$ \\
\hline
\end{tabular}

when $n_{\text {right }}$ is small, whereas increases are more readily detected when $n_{\text {left }}$ is small and $n_{\text {right }}$ is large. This constellation came along with a significant three-way interaction, $F(2,102)=9.273, \eta^{2}=.154, p<.001$. A $p$ change main effect, $F(2,102)=14.436, \eta^{2}=.221$, $p<.001$, is due to reduced accuracy for no-change trials (see Fig. 3 and Table 3). Apparently, judges were reluctant to classify sample pairs as equal, given the small number of only 16 (out of 64) no-change trials.

\section{General discussion}

To summarize, several experiments provided convergent evidence for a distinct and stable pattern of anomalies in the detection of change. Whether the task referred to the proportion of a graphical feature in a meaning-free psychophysical setting, to proportions of smiling faces in a meaningful social setting, to changes of successively or simultaneously presented samples, change judgments reflected the same pattern of systematic biases. Increasing proportions were detected more readily when sample sizes also increased but were hardly or incorrectly recognized when sample size decreased. Conversely, the detection of decreasing proportions was facilitated by a decrease in sample size and impaired by an increase in sample size. When no changes had occurred, false alarms corresponded to the change in sample size. These biases were so strong that changes in $n$ often fully overrode the actual changes in $p$.

In further studies to be reported in separate articles, we continued to find convergent evidence with other geometric stimuli ( $\equiv$ and o) and even when feedback was provided about the correctness of change judgments and when judges were warned of the misleading impact of $n$ (Fiedler \& Schott,

${ }^{6}$ Change assessment in this experiment was inferred from the sensitivity of repeated teachers' judgments to actually occurring changes in student performance rather than direct judgments of change.
2015). Thus, the anomalies persisted at a high level of effect sizes after participants were explicitly informed that errors in change judgment were mainly due to $n$ increases leading to erroneous "increase" responses and $n$ decreases leading to erroneous "decrease" responses.

Moreover, we found even more radical influences of $n$ changes on the assessment of $p$-changes in the very task setting that we have repeatedly used to illustrate the phenomenon, namely, teachers' assessment of changing student performance in a simulated classroom setting (Fiedler, Woellert, Tauber, \& Hess, 2015). ${ }^{6}$ When the correctness rates of subsets of the 12 students increased and decreased from one lesson to another, this was only recognized if the respective students' cooperation rate (i.e., how often they raised their hand and got a chance to respond) changed in the same direction. A precondition for teachers (participants) to recognize increases (decreases) in student performance - that is, in $p$ (correct response) - was that the sample size of student responses $n$ increased (decreased) in the same direction.

How can this robust pattern of anomalies in change detection be explained? - Let us first of all rule out a trivial account in terms of a sheer misunderstanding of the inference task. Despite the explicit instruction to judge $p$-changes in the latent world, as distinguished from changes in the number of focal features in the manifest samples, we did not deny the possibility that participants may have not followed the explicit task instructions.

A number of findings render such an account unlikely. Regression analyses (across trials within all individual judges) revealed a systematic impact of actual $p$-changes beyond the independent impact of $n$-changes on change judgments. The most compelling evidence came from Experiment 3, which included both equal- $n$ and unequal- $n$ trials. The robust anomalies were confined to unequal- $n$ trials. On equal- $n$ trials, $p$ changes were assessed accurately and independently of whether the corresponding change in the absolute number of focal features (i.e., in the numerator) was large (when $n_{\text {left }}=$ $n_{\text {right }}=16$ ) or small (when $n_{\text {left }}=n_{\text {right }}=8$ ). This finding highlights the fact that a summation rule alone cannot explain the 
results, because judgments were not only sensitive to $n$ but also to $p$. Thus, judges are in principle able to assess $p$ in a sample of a given size. However, while this ability is apparent for equal- $n$ comparisons, it is impaired when the comparison context obscures sample size, that is, when a comparison sample mimics too large or too small a sample size.

Second, the latter finding is also at variance with a simple Bayesian account, which says that the same high or low proportion observed a large sample (e.g., 12 positive and 4 negative outcomes) provides stronger evidence that $p$ is actually high (low) than in a small sample (6 positive and 2 negative). According to this rule, the same proportional shift should appear stronger when $n_{\text {left }}=n_{\text {right }}=16$ than when $n_{\text {left }}=n_{\text {right }}=8$, which was not the case.

A simple Bayesian account, according to which judges have an intuitive understanding of the normative meaning of sample size, is particularly incompatible with the elimination of the strong anomalies when samples were presented in a probability format (as normalized percentages). It is hard to see why such Bayesian implications should not hold when $p$ changes are presented on a percentage scale.

And third, literal denominator neglect alone cannot explain the present findings either, because judgments were sensitive to $p$, either independently of $n$ or exclusively when $n$ did not vary. However, a more refined version of denominator neglect comes very close to our explanation of why an inductive learning algorithm cannot deal with changing $n$. Throughout this article, we have emphasized that the inductive assessment of $p$ from an experienced sample must be calibrated for $n$, which has to be known beforehand, because every elementary observation or subset of 2,3 , or 4 observations has a stronger impact on the $p$ estimate when $n$ is small rather than large. The weight of a single elementary observation is .5 for $n=2, .2$ for $n=5, .1$ for $n=10, .05$ for $n=20$, or, in general, $1 / n$. Therefore, the induction algorithm process must somehow adapt its sensitivity - that is, the unit weight given to new observations - as $n$-changes.

To explain the entire pattern of results, we only have to assume that the sensitivity (i.e., the unit weight given to elementary observations) of the preceding sample to some extent carries over to the subsequent sample. This assumption is sufficient to explain that a small $n$ in the preceding sample should sensitize the induction process, letting the numerator (i.e., the number of focal features) of a subsequent sample appear larger (due to an enhanced weight given to elementary events). Conversely, a large $n$ in the preceding sample should desensitize the induction process, letting the subsequent numerator increments appear smaller. As a consequence, increases (decreases) in $n$ should facilitate the assessment of increasing (decreasing) $p$, but counteract the assessment of decreasing (increasing) $p$.

Note that this account does not assume a total neglect of the denominator; judgments are not insensitive to the sample proportions proper. The impact of $p$-changes on change judgments that was obtained independently of the impact of $n$ changes, implies that the cognitive algorithm is not fully restricted to counting the number of focal features (i.e., the numerator) but it is also somehow influenced by the size of the sample (i.e., the denominator). However, because $n$ is variable, initially unknown and gradually emerging, the algorithm cannot rely on the computation of a ratio whose denominator is indeterminate. All the algorithm can do, instead, is to adjust the increment or decrement encoded for an elementary observation to the sampling context. Being calibrated to a small $n$ on the preceding sample, the online experience of increments and decrements in a subsequent sample should be given more weight than when the preceding $n$ has been large.

We refrain from any attempt to quantify this dynamic calibration process, which may vary as a function of many factors. All we assume is that judges manage to adjust to some degree the weight given to elementary stimuli to some rudimentary measure of the size or volume of the sample. The twofold sensitivity we have consistently obtained for $p$-changes as well as $n$-changes implies that the increments or decrements coded for elementary observations of the focal feature increased when the current sample was absolutely small (rather than large) and when the preceding sample was relatively smaller (rather than larger) than the current sample.

Such a simple assumption can explain the entire pattern of evidence obtained across all experiments. In particularly, it can explain why anomalies disappeared in Experiment 3, when sample size remained constant. And, by the same token, it makes perfect sense that the anomalies were eliminated, in spite of explicitly provided $n$, when proportional changes were presented on a normalized percentage scale, which renders calibration irrelevant.

The present approach goes beyond several other, seemingly related theoretical concepts that have been reviewed in the introduction. Neither the notion of numerical magnitude priming (Oppenheimer et al., 2008; Wong \& Kwong, 2000) nor the concepts of ratio bias or denominator neglect (Denes-Raj \& Epstein, 1994) per se can explain the format dependence of the reported anomalies. Our findings suggest that judges are not simply insensitive to ratios (proportions) and denominators (sample sizes). Rather, in situations when changing denominators call for dynamic recalibration of the weight given to elementary observations (i.e., when samples are experienced), the size of the preceding sample seems to affect the calibration of the sensitivity for the subsequent sample. However, it may be premature and unjustified to attribute such a miscalibration effect to cognitive deficits or lack of rationality. Since the ultimate $n$ of experienced samples is rarely known beforehand, the induction process cannot be precisely calibrated to $1 / n$ and, apparently, the size of the preceding sample serves as a proxy for calibration under uncertainty. 
Consistent with such an analysis of the inductive-encoding dilemma, the anomalies were confined to experienced samples. They were fully eliminated in the probability mode condition when all sample changes were indicated in a normalized percentage scale so that the inductive inference process was cut short and calibration (of increments or decrements encoded for elementary raw events) was unnecessary. Even when samples were experienced, the anomalies disappeared on equal- $n$ trials, when the calibration of a current sample could not be led astray by a different $n$ on the preceding sample. It also makes sense that anomalies do occur in the cardinal-frequency condition, in which numerical differences in the numerator are contingent on changes in the denominator, unlike probability format or equal- $n$ conditions.

Our manipulation of presentation formats may be reminiscent of a large body of recent research on the "descriptionexperience gap" in decision making research (Hau et al., 2008; Hertwig \& Erev, 2009), but again our findings go beyond this literature. So far, this prominent research has focused almost exclusively on the overweighting of rare events in descriptive mode, which turns into underweighting when samples are experienced (Hertwig \& Erev, 2009). Another debate revolves around whether sampling biases can account for the seeming format effect (Rakow et al., 2008). The present findings serve to widen the domain of the descriptionexperience gap, which is not confined to preference reversals for small- $p$ lotteries but strongly moderates the detection of change across the entire range of $p$ values. Moreover, because the same sampling algorithm was used to generate descriptive and experienced samples, our findings cannot reflect sampling error, that is, discrepancies between experienced samples and described population parameters.

One completely new feature of the present paradigm is that judgments call for inferences from sample proportions to (population) parameters of the latent world. Virtually all previous research - from Peterson and Beach (1967) work on the intuitive statistician to recent research on ratio bias, denominator neglect and sample-size bias - is concerned with the precise assessment of sample proportions. In contrast, we have defined change detection as an inference task, which might itself be sensitive to sample size. Because this inferential component is essential for change detection in reality - in school classes, politics, survey research, or the stock market - we believe that our paradigm affords a more suitable experimental analogue than merely psychophysical studies of sample proportions.

The term anomalies that we have used throughout this manuscript for the intrusion of sample size in change detection was never intended as a label for irrational or dysfunctional behavior. It was only used as a descriptive term to denote a category mistake on a specific task: Change judgments were systematically influenced by $n$-changes on a task that called for the assessment of $p$-changes. If such an anomaly is to be called "irrational" depends not only on one's definition of irrationality (McKenzie, 2003) but also on a careful analysis of the problem structure (Neth, Sims, \& Gray, 2015). One could indeed imagine that using $n$-change as an easily detectable and often redundant cue for the assessment of $p$-changes can be quite functional under several task settings. However, in other task settings, when it is essential to distinguish between absolute and relative size, the anomalies in change detection can be clearly dysfunctional and irrational (i.e., working against the rationale of the task).

Because such unwanted consequences cannot be denied, we believe that in addition to all theoretical implications, the present results have immediate practical implications as well. The school class scenario we have used for illustration throughout this paper provides a prominent example (cf. Fiedler et al., 2002). Fair grading and accurate achievement assessment are of interest to everybody; it is crucial for valid feedback and selection decisions. Yet, our findings strongly suggest that teachers' evaluations may confound quality and quantity of student performance. The work of Price et al. (2006) on the impact of group size on risk assessment provides another intriguing example. Similar points could be made for the impact of exposure frequency in the media on political evaluations, consumer products, or stereotyped groups. In all these applied domains, successful debiasing and interventions depend crucially on the ability to assess proportions independently of absolute sample size. The present findings suggest that task design and presentation format should have a strong impact on the accuracy and achievement of change detection.

Author Note The research underlying this paper was supported by grant 1020-303.4/2008 of the German-Israeli Foundation of Scientific Research and Development (GIF).

\section{Appendix: Full translated instruction text}

The present investigation is concerned with the detection of changes in the environment. It is sometimes not easy to distinguish genuine changes from random fluctuations. Has the weather or the climate really changed, or does it just reflect normal variation? Did the number of visitors in a pub decline, or is it the number of people just low at the moment? Are the new beginning semesters more stupid or smarter than the old ones, or did nothing change but random variation?

To study the sensitivity to such distinctions, we have devised the following task. On every trial of a longer series, you will be presented with a sample in which two symbols, and \&, appear at variable frequencies. The sample is drawn randomly from a universe of 100 symbols, in which the occurrence rate of the two symbols is determined. 
The question on every trial is: Is the current sample drawn from the same universe as the preceding one, or is the universe from which the new sample is drawn different from the preceding one? That is, has the environment changed?

Thus, the task to distinguish "genuine" changes from mere random fluctuations is defined clearly: Was the last samples drawn from the same "world" as the second-last? That is, could the changing percentage rate of the two symbols arise from random variation alone, as a new sample was drawn from the same "world"? Or must the "world" have changed, that is, must the last sample stem from a changed universe, in which the proportion of the two symbols is no longer the same?

The critical question on every trial always focuses on one of the two symbols, namely, the symbol. The question is whether the "world," or universe, has remained the SAME, or whether there was an INCREASE or DECREASE in the proportion of the critical symbol. Please respond by pressing one of the corresponding three response keys. Then, a short moment later, the next trial starts on which you will again be asked if this time the "world" has changed or not.

First of all, however, you will see two demonstration trials (1)

You have now experienced two demonstration trials purported to familiarize you with the task ...

Please make an attempt to provide as many correct responses as possible. Beware of the fact that the correct response is determined, because each sample is in fact drawn either from the same "world," or universe, or from one that has changed.

PLEASE KEEP THE FOLLOWING IN MIND:

EVEN WHEN THE WORLD DOES NOT CHANGE, RATHER STRONG FLUCTUATIONS MAY OCCUR SIMPLY BECAUSE SAMPLES DRAWN FROM THE SAME WORLD CAN VARY MORE THAN ONE MIGHT EXPECT. IN OTHER WORDS, IF YOU ARE UNCERTAIN, YOU MAY BE BETTER OFF IF YOU RESPOND "Same" AND ONLY INDICATE A CHANGE IF THE OBSERVED CHANGE IS ACTUALLY MARKED.

At the end you will be shown in a clearly arranged table how your responses across all trials match with the correct solutions.

Enjoy your task.

\section{References}

Allan, L. (1980). A note on measurement of contingency between two binary variables in judgment tasks. Bulletin of the Psychonomic Society, 15(3), 147-149. doi:10.3758/BF03334492

Allan, L., \& Jenkins, H. (1983). The effect of representations of binary variables on judgment of influence. Learning and Motivation, 14(4), 381-405. doi:10.1016/0023-9690(83)90024-3
Brown, S. D., \& Steyvers, M. (2009). Detecting and predicting changes. Cognitive Psychology, 58(1), 49-67. doi:10.1016/j.cogpsych.2008. 09.002

Chinnis, J. R., \& Peterson, C. R. (1970). Nonstationary processes and conservative inference. Journal of Experimental Psychology, 84(2), 248-251. doi:10.1037/h0029068

Dechêne, A., Stahl, C., Hansen, J., \& Wänke, M. (2010). The truth about the truth: A meta-analytic review of the truth effect. Personality and Social Psychology Review, 14(2), 238-257.

Denes-Raj, V., \& Epstein, S. (1994). Conflict between intuitive and rational processing: When people behave against their better judgment. Journal of Personality and Social Psychology, 66(5), 819829. doi:10.1037/0022-3514.66.5.819

Denes-Raj, V., Epstein, S., \& Cole, J. (1995). The generality of the ratiobias phenomenon. Personality and Social Psychology Bulletin, 21(10), 1083-1092. doi:10.1177/01461672952110009

Estes, W. K. (1976). The cognitive side of probability learning. Psychological Review, 83(1), 37.

Fiedler, K. (1988). The dependence of the conjunction fallacy on subtle linguistic factors. Psychological Research, 50, 123-129.

Fiedler, K. (1996). Explaining and simulating judgment biases as an aggregation phenomenon in probabilistic, multiple-cue environments. Psychological Review, 103(1), 193-214. doi:10.1037/0033295X.103.1.193

Fiedler, K. (2000a). Beware of samples! A cognitive-ecological sampling approach to judgment biases. Psychological Review, 107(4), 659676. doi:10.1037/0033-295X.107.4.659

Fiedler, K. (2000b). Illusory correlations: A simple associative algorithm provides a convergent account of seemingly divergent paradigms. Review of General Psychology, 4(1), 25-58. doi:10.1037/10892680.4.1.25

Fiedler, K., \& Krueger, J. I. (2012). More than an artifact: Regression as a theoretical construct. In J. I. Krueger (Ed.), Social judgment and decision making (pp. 171-189). New York, NY: Psychology Press.

Fiedler, K., \& Schott, M. (2015). Metacognitive myopia and insensitivity to feedback in the detection of change. Unpublished research, University of Heidelberg, Heidelberg, Germany.

Fiedler, K., Freytag, P., \& Meiser, T. (2009). Pseudocontingencies: An integrative account of an intriguing cognitive illusion. Psychological Review, 116(1), 187-206. doi:10.1037/a0014480

Fiedler, K., Walther, E., Freytag, P., \& Plessner, H. (2002). Judgment biases in a simulated classroom-A cognitive-environmental approach. Organizational Behavior and Human Decision Processes, 88(1), 527-561. doi:10.1006/obhd.2001.2981

Fiedler, K., Walther, E., \& Nickel, S. (1999). The auto-verification of social hypotheses: Stereotyping and the power of sample size. Journal of Personality and Social Psychology, 77(1), 5-18. doi:10. 1037/0022-3514.77.1.5

Fiedler, K., Woellert, F., Tauber, B., \& Hess, P. (2015). Anomalies in detecting performance changes in a simulated classroom environment. Unpublished research, University of Heidelberg, Heidelberg, Germany.

Fox, C. R., \& Hadar, L. (2006). "Decisions from experience" = sampling error + prospect theory: Reconsidering Hertwig, Barron, Weber \& Erev (2004). Judgment and Decision Making, 1(2), 159-161.

Gigerenzer, G. (1991). How to make cognitive illusions disappear: Beyond "heuristics and biases.". In W. Stroebe \& M. Hewstone (Eds.), European Review of Social Psychology (Vol. 2, pp. 83115). Chichester: Wiley.

Gigerenzer, G. (1994). Why the distinction between single-event probabilities and frequencies is relevant for psychology and vice versa. In G. Wright \& P. Avion (Eds.), Subjective probability (pp. 129-162). New York: Wiley.

Gigerenzer, G., \& Hoffrage, U. (1995). How to improve Bayesian reasoning without instruction: Frequency formats. Psychological Review, 102(4), 684-704. doi:10.1037/0033-295X.102.4.684 
Hamilton, D. L., Dugan, P. M., \& Trolier, T. K. (1985). The formation of stereotypic beliefs: Further evidence for distinctiveness-based illusory correlations. Journal of Personality and Social Psychology, 48(1), 5-17.

Hamilton, D. L., \& Gifford, R. K. (1976). Illusory correlation in interpersonal perception: A cognitive basis of stereotypic judgment. Journal of Experimental Social Psychology, 12, 392-407.

Hau, R., Pleskac, T. J., Kiefer, J., \& Hertwig, R. (2008). The descriptionexperience gap in risky choice: The role of sample size and experienced probabilities. Journal of Behavioral Decision Making, 21, 493-518. doi:10.1002/bdm.598

Hertwig, R., Barron, G., Weber, E., \& Erev, I. (2004). Decisions from experience and the effect of rare events in risky choice. Psychological Science, 15(8), 534-539.

Hertwig, R., \& Erev, I. (2009). The description-experience gap in risky choice. Trends in Cognitive Sciences, 13(12), 517-523. doi:10. 1016/j.tics.2009.09.004

Hertwig, R., Herzog, S. M., Schooler, L. J., \& Reimer, T. (2008). Fluency heuristic: A model of how the mind exploits a by-product of information retrieval. Journal of Experimental Psychology: Learning, Memory, and Cognition, 34(5), 1191-1206. doi:10.1037/a0013025

Hilbert, M. (2012). Toward a synthesis of cognitive biases: How noisy information processing can bias human decision making. Psychological Bulletin, 138(2), 211-237. doi:10.1037/a0025940

Hoffrage, U., \& Gigerenzer, G. (1998). Using natural frequencies to improve diagnostic inferences. Academic Medicine, 73(5), 538-540. doi:10.1097/00001888-199805000-00024

Inhelder, B., \& Piaget, J. (1958). The growth of logical thinking from childhood to adolescence. New York: Basic Books.

Kutzner, F., Freytag, P., Vogel, T., \& Fiedler, K. (2008). Base-rate neglect as a function of base rates in probabilistic contingency learning. Journal of the Experimental Analysis of Behavior, 90, 23-32.

Kutzner, F., Vogel, T., Freytag, P., \& Fiedler, K. (2011). A robust classic: Illusory correlations are maintained under extended operant learning. Experimental Psychology, 58(6), 443-453. doi:10.1027/16183169/a000112

Massey, C., \& Wu, G. (2005). Detecting regime shifts: The causes of under- and overreaction. Management Science, 51(6), 932-947.

McKenzie, C. M. (2003). Rational models as theories - not standardsof behavior. Trends in Cognitive Sciences, 7(9), 403-406. 10.1016/ S1364-6613(03)00196-7

Moore, D. A., \& Healy, P. J. (2008). The trouble with overconfidence. Psychological Review, 115(2), 502-517. doi:10.1037/0033-295X. 115.2.502

Neth, H., Sims, C. R., \& Gray, W. D. (2015). Rational task analysis: A methodology to benchmark bounded rationality. Minds and Machines. doi:10.1007/s11023-015-9368-8

Oppenheimer, D. M., LeBoeuf, R. A., \& Brewer, N. T. (2008). Anchors aweigh: A demonstration of cross-modality anchoring and magnitude priming. Cognition, 106(1), 13-26. doi:10.1016/j.cognition. 2006.12.008

Perales, J., Catena, A., Shanks, D., \& González, J. (2005). Dissociation between judgments and outcome-expectancy measures in covariation learning: A signal detection theory approach. Journal of Experimental Psychology: Learning, Memory, and Cognition, 31(5), 1105-1120. doi:10.1037/0278-7393.31.5.1105

Peterson, C. R., \& Beach, L. R. (1967). Man as an intuitive statistician. Psychological Bulletin, 68(1), 29-46. doi:10.1037/h0024722

Price, P. C. (2001). A group size effect on personal risk judgments: Implications for unrealistic optimism. Memory \& Cognition, 29(4), 578-586. doi:10.3758/BF03200459

Price, P. C., Smith, A. R., \& Lench, H. C. (2006). The effect of target group size on risk judgments and comparative optimism: The more, the riskier. Journal of Personality and Social Psychology, 90(3), 382-398. doi:10.1037/0022-3514.90.3.382

Rakow, T., Demes, K. A., \& Newell, B. R. (2008). Biased samples not mode of presentation: Re-examining the apparent underweighting of rare events in experience-based choice. Organizational Behavior and Human Decision Processes, 106(2), 168-179.

Rapoport, A., Stein, W. E., \& Burkheimer, G. J. (1979). Response models for detection of change. Dordrecht: D. Reidel.

Reyna, V. F., \& Brainerd, C. J. (2008). Numeracy, ratio bias, and denominator neglect in judgments of risk and probability. Learning and Individual Differences, 18(1), 89-107. doi:10.1016/j.lindif.2007.03. 011

Robinson, G. H. (1964). Continuous estimation of a time-varying probability. Ergonomics, 7(1), 7-21. doi:10.1080/00140136408930721

Shanks, D. R. (1985). Continuous monitoring of human contingency judgment across trials. Memory \& Cognition, 13, 158-167.

Shanks, D. R. (1987). Acquisition functions in contingency judgment. Learning and Motivation, 18, 147-166.

Shanks, D. R., \& Dickinson, A. (1991). Instrumental judgment and performance under variations in action outcome contingency and contiguity. Memory \& Cognition, 19, 353-360.

Sloman, S. A., Over, D., Slovak, L., \& Stibel, J. M. (2003). Frequency illusions and other fallacies. Organizational Behavior and Human Decision Processes, 91, 296-309.

Slovic, P., Monahan, J., \& MacGregor, D. G. (2000). Violence risk assessment and risk communication: The effects of using actual cases, providing instruction, and employing probability versus frequency formats. Law and Human Behavior, 24(3), 271. doi:10.1023/ A: 1005595519944

Smith, A. R., \& Price, P. C. (2010). Sample size bias in the estimation of means. Psychonomic Bulletin \& Review, 17(4), 499-503. doi:10. 3758/PBR.17.4.499

Smith, E. R. (1991). Illusory correlation in a simulated exemplar-based memory. Journal of Experimental Social Psychology, 27, 107-123.

Smolensky, P. (1988). On the proper treatment of connectionism. Behavioral and Brain Sciences, 11, 1-74.

Speekenbrink, M., Twyman, M. A., \& Harvey, N. (2012). Change detection under autocorrelation. In N. Myake, D. Peebles, \& R. P. Cooper (Eds.), Proceedings of the 34th Annual Conference of the Cognitive Science Society (pp. 1001-1006). Austin, TX: Cognitive Science Society.

Ungemach, C., Chater, N., \& Stewart, N. (2009). Are probabilities overweighted or underweighted when rare outcomes are experienced (rarely)? Psychological Science, 20(4), 473-479. doi:10. 1111/j.1467-9280.2009.02319.x

Van Rooy, D., Van Overwalle, F., Vanhoomissen, T., Labiouse, C., \& French, R. (2003). A recurrent connectionist model of group biases. Psychological Review, 110, 536-563.

Wasserman, E. A., Dorner, W. W., \& Kao, S. F. (1990). Contributions of specific cell information to judgments of interevent contingency. Journal of Experimental Psychology: Learning, Memory, and Cognition, 16(3), 509-521. doi:10.1037/0278-7393.16.3.509

White, P. (2009). Accounting for occurrences: An explanation for some novel tendencies in causal judgment from contingency information. Memory \& Cognition, 37(4), 500-513. doi:10.3758/MC.37.4.500

Wong, K. F. E., \& Kwong, J. Y. Y. (2000). Is $7300 \mathrm{~m}$ equal to $7.3 \mathrm{~km}$ ? Same semantics but different anchoring effects. Organizational Behavior and Human Decision Processes, 82, 314-333.

Zajonc, R. B. (2001). Mere exposure: A gateway to the subliminal. Current Directions in Psychological Science, 10(6), 224-228. doi: $10.1111 / 1467-8721.00154$ 